\title{
Methyltransferase inhibitors restore SATB1 protective activity against cutaneous T cell lymphoma in mice
}

\author{
Carly M. Harro, ${ }^{1,2,3}$ Jairo Perez-Sanz,, Tara Lee Costich, ${ }^{1}$ Kyle K. Payne, ${ }^{1}$ Carmen M. Anadon, ${ }^{1}$ Ricardo A. Chaurio, ${ }^{1}$ Subir Biswas, ${ }^{1}$ \\ Gunjan Mandal, ${ }^{1}$ Kristen E. Rigolizzo, ${ }^{1}$ Kimberly B. Sprenger, ${ }^{1}$ Jessica A. Mine, ${ }^{1}$ Louise C. Showe, ${ }^{4}$ Xiaoqing Yu, ${ }^{5}$ Kebin Liu, ${ }^{6}$ \\ Paulo C. Rodriguez, ${ }^{1}$ Javier Pinilla-Ibarz, ${ }^{7}$ Lubomir Sokol, ${ }^{7}$ and Jose R. Conejo-Garcia ${ }^{1,8}$ \\ 'Department of Immunology, H. Lee Moffitt Cancer Center \& Research Institute, Tampa, Florida, USA. ²Department of Cell Biology, Microbiology, and Molecular Biology, and ${ }^{3}$ Cancer Biology PhD Program, \\ College of Arts and Sciences, University of South Florida, Tampa, Florida, and H. Lee Moffitt Cancer Center \& Research Institute, Tampa, Florida, USA. ${ }^{4}$ Molecular \& Cellular Oncogenesis Program, The Wistar \\ Institute, Philadelphia, Pennsylvania, USA. ${ }^{5}$ Biostatistics and Bioinformatics, H. Lee Moffitt Cancer Center \& Research Institute, Tampa, Florida, USA. ${ }^{6}$ Department of Biochemistry and Molecular Biology, \\ Medical College of Georgia, Augusta, Georgia, USA. Department of Malignant Hematology, and ${ }^{8}$ Department of Gynecologic Oncology, H. Lee Moffitt Cancer Center \& Research Institute, Tampa, Florida, USA.
}

\begin{abstract}
Cutaneous T cell lymphoma (CTCL) has a poorly understood etiology and no known cure. Using conditional knockout mice, we found that ablation of the genomic organizer special AT-rich sequence-binding protein 1 (Satb1) caused malignant transformation of mature, skin-homing, Notch-activated $C D 4^{+}$and $C D 8^{+} \mathrm{T}$ cells into progressively fatal lymphoma. Mechanistically, Satb1 restrained Stat 5 phosphorylation and the expression of skin-homing chemokine receptors in mature T cells. Notably, methyltransferase-dependent epigenetic repression of SATB1 was universally found in human Sézary syndrome, but not in other peripheral T cell malignancies. H3K27 and H3K9 trimethylation occluded the SATB1 promoter in Sézary cells, while inhibition of SUV39H1/2 methyltransferases (unlike EZH2 inhibition) restored protective SATB1 expression and selectively abrogated the growth of primary Sézary cells more effectively than romidepsin. Therefore, inhibition of methyltransferases that silence SATB1 could address an unmet need for patients with mycosis fungoides/Sézary syndrome, a set of incurable diseases.
\end{abstract}

\section{Introduction}

Peripheral T cell lymphomas (PTCLs) represent a collection of aggressive disorders with unfavorable outcome that account for approximately $12 \%$ of lymphoid tumors worldwide (1). Cutaneous $\mathrm{T}$ cell lymphoma (CTCL) belongs to a heterogeneous group of $\mathrm{T}$ cell lymphomas characterized by a cutaneous infiltration of malignant $\mathrm{T}$ cells. No curative therapies are currently available for advanced mycosis fungoides and its leukemic variant, Sézary syndrome, which are the most common forms of CTCL, with an annual incidence of approximately 0.5 per 100,000 (2). NOTCH1 is overexpressed in CTCL in a stage-dependent manner (3), and recent studies have suggested a role for other potential oncogenic drivers, including TP53, RB1, PTEN, and DNMT3A (4). The similarities and differences in the molecular underpinnings of these heterogeneous diseases remain poorly understood, and seminal

Authorship note: CMH and JPS contributed equally to this work. Conflict of interest: KL holds the US patent 10,577,371 B2, "Small Molecule Histone Methyltransfease SUV39H1 Inhibitor and Uses Thereof." JPI receives funding from TC therapeutics, MEI, and Sunesis, and consulting fees from Janssen, Abbvie, TC therapeutics, Novartis, and AstraZeneca. LS is an advisor for Kyowa-Kirin, Inc and Kymera Therapeutics, and receives clinical research funding from EUSA Pharma, LLC. JRCG receives funding and consulting fees from Anixa Biosciences and Compass Therapeutics, and consulting fees from Leidos.

Copyright: () 2021, American Society for Clinical Investigation. Submitted: December 12, 2019; Accepted: November 25, 2020;

Published: February 1, 2021

Reference information: J Clin Invest. 2021;131(3):e135711.

https://doi.org/10.1172/JCI135711 genomic analyses are restricted to a handful of patients in the pathogenesis of mycosis fungoides/Sézary syndrome (4). Informative animal models that reflect multi-step oncogenesis and cover a broader spectrum of CTCL are therefore needed to test more effective treatments and identify potentially novel therapeutic targets. Lack of mechanistic understanding and the subsequent absence of curative interventions have led to poor prognoses and serious deterioration in the quality of life for patients with advanced CTCL disease. A better understanding of the pathogenesis of this disease is urgently needed to design new treatments that improve both quality of life and survival outcome.

The chromatin organizer special AT-rich sequence-binding protein 1 (SATB1) plays essential roles in apoptosis, cell invasion, metastasis, and proliferation of cancer cells (5). In addition, a dynamic SATB1 expression pattern controls the phenotype of tumor-associated dendritic cells (6) and cytotoxic T cells (7), which in coordination determine malignant progression. In addition to cancer, SATB1 modulates the phenotype of regulatory T cells (8) as well as the epigenetic and transcriptional pathways required for hematopoietic stem cell division and self-renewal (9). Interestingly, recent reports suggest that SATB1 could be downregulated in mycosis fungoides (10), although epigenetic silencing mechanisms via histone modification and the consequences of SATB1 downregulation remain uninvestigated. Using a triple-transgenic mouse for concurrent ablation of Satb1 and activation of Notch1 signaling specifically in mature $\mathrm{T}$ cells, we generated a reproducible model of progressive fatal T cell lymphoma/leukemia. This system provides possible insight into the role of $S A T B 1$ as a sup- 
pressor gene in CTCL and allowed us to identify potential interventions to restore SATB1 function in primary malignant $\mathrm{T}$ cells.

\section{Results}

Ablation of Satb1 cooperates with Notch1 overexpression to cause T cell leukemia lymphoma. We generated a triple-transgenic (CD11c $c^{\mathrm{Cre}}$ Satb $1^{f / f l} \operatorname{Rosa} 26^{\text {NI-ICD }}$ ) mouse model to bypass the requirement of Satb1 for Notch1 expression in bone marrow dendritic cells (BMDCs) (6). In this system, CD11c expression triggers Satb1 ablation while simultaneously driving the expression of the Notch1 intracellular domain ( $\left.\mathrm{N}^{\mathrm{ICD}}\right)$ (11). Mice developed normally as neonates but exhibited a progressive increase in $\mathrm{T}$ cell numbers in peripheral blood. Unexpectedly, all triple-transgenic mice, but not $C D 11 c^{C r e} S a t b 1^{f / f l}$ or $C D 11 c^{C r e} R o s a 26^{N 1-I C D}$ littermates, developed a decrease in activity and ruffled fur after approximately 10 weeks, followed by tensional ascites and difficulty breathing within the next 2 weeks, when the mice became moribund. Compared with littermates, 8- to 10-week-old triple-transgenic mice showed prominent splenomegaly and hepatomegaly, along with pronounced adenopathy and effacement of normal architecture in the kidneys (Figure 1, A, C, and D). Triple-transgenic mice demonstrated significantly decreased survival compared with $C D 11 c^{\mathrm{Cr}}$ $S a t b 1^{f l / f l}$ and $C D 11 c^{C r e}$ Rosa $26^{\text {N1-ICD }}$ littermates (Figure 1B).

$\mathrm{T}$ cell accumulation of tumor-bearing $\mathrm{CD} 11 \mathrm{c}^{\mathrm{Cre}}$ mice presented no detectable expansion of NK or $\gamma \delta$ T cells in the peripheral blood (Figure 2A). Although the phenotype was driven by a CD11c Cre transgene, flow cytometric analyses of Satb1-excised cells (based on green fluorescence linked to Notch1 expression) revealed progressive accumulation of $\mathrm{GFP}^{+} \mathrm{CD}^{+} \mathrm{CD}^{+} \mathrm{T}$ cells in peripheral blood, which is consistent with the expression of CD11c in a subset of $\mathrm{CD}^{+} \mathrm{T}$ cells $(12,13)$ (Figure 2B). Accordingly, the disease evolved from the accumulation of a population of $\mathrm{CD}^{+} \mathrm{T}$ cells in peripheral blood, which progressively turned into $\mathrm{CD} 4^{+} \mathrm{CD} 8^{+}$ double-positive $\mathrm{T}$ lymphocytes and became the most abundant $\mathrm{T}$ cell subset after approximately 8 weeks (Figure $2 \mathrm{C}$ ). In contrast, although $S a t b 1^{+} C D 11 c^{C r e}$ Rosa $26^{N 1-I C D}$ littermates also showed some progressive expansion of Notch-knockin $\left(\mathrm{GFP}^{+}\right) \mathrm{CD}^{+} \mathrm{T}$ cells (Figure 2D), no mice in this group exhibited accumulation of $\mathrm{CD} 4^{+} \mathrm{CD} 8^{+}$lymphocytes at identical temporal points or signs of disease at any time (Figure $2 \mathrm{E}$ ). Together, these results indicate that Notch1 overexpression in a subset of mature CD11 $\mathrm{c}^{+} \mathrm{CD} 8^{+} \mathrm{T}$ cells alone is not sufficient to drive malignant progression. However, additional ablation of Satb1 results in progressive and lethal accumulation of $\mathrm{T}$ cells that become $\mathrm{CD} 4^{+} \mathrm{CD} 8^{+}$double-positive lymphocytes at terminal disease stages.

Ablation of Satb1 induces malignant lymphocytic expansion by promoting phosphorylation of Notch-induced Stat5. Microscopic examination of skin harvested from $C D 11 c^{C r e} S a t b 1^{f / f l} R o s a 26^{N 1-I C D}$ mice showed $\mathrm{CD}^{+} \mathrm{T}$ cell infiltrates that were stained as described previously (14). $\mathrm{CD}^{+} \mathrm{T}$ cell infiltrates densely clustered within the dermis, with individual lymphocytic cells present within the epidermis in the majority of CD11c Cre Satb1 $1^{f l / f l}$ Rosa2 $6^{\mathrm{N1}-\mathrm{ICD}}$ mice examined (Figure 3A and Supplemental Figure 1; supplemental material available online with this article; https://doi.org/10.1172/ JCI135711DS1). Quantification via positive pixel count of $\mathrm{CD}^{+} \mathrm{T}$ cells showed significant infiltration in CD11 $c^{C r e} S a t b 1^{f / f l} R o s a 26^{N 1-I C D}$, compared with single-genotype littermates (Figure 3B). Examina- tion of peripheral blood smears at approximately 8 weeks confirmed the expansion of immature lymphocytic cells with larger nuclei and prominent nucleoli (Figure 3C), matching the morphology of malignant lymphocytes.

To understand how ablating Satb1 transforms the mild expansion of $\mathrm{CD}^{+} \mathrm{T}$ cells observed in $C D 11 c^{\mathrm{Cr} r} \mathrm{Rosa} 26^{\mathrm{N1-ICD}}$ mice into a fatal lymphocytic expansion, we focused on differences in Stat signaling. As shown in Figure 3D, knocking in Notch1 in CD11 $\mathrm{c}^{+} \mathrm{CD} 8^{+}$ $\mathrm{T}$ cells resulted in the upregulation of total Stat $5 \alpha$, while Satb1 ablation alone had no effect on Stat 5 expression. However, we only observed high levels of Stat 5 phosphorylation upon combined Satb1 ablation and Notch1 (N1 ${ }^{\mathrm{ICD}}$ ) overexpression (Figure 3D).

To elucidate the mechanism driving Stat5 phosphorylation upon Satb1 deletion, we focused on cytokine secretion by transgenic T cells. We found that $C D 11 c^{C r e} S a t b 1^{f / f l} R o s a 26^{N-I C D} \mathrm{~T}$ cells produce an approximately 7 -fold increase in IL-2, compared with their counterparts from $\mathrm{Cre}^{-}$littermates (Figure 3E). These data indicate that $S a t b 1$ ablation derepresses IL-2 production by T cells. Therefore, Notch1 signaling results in Stat 5 overexpression. When Satb1 deletion derepresses IL-2 production in the same lymphocytes, upregulated Stat5 is more actively phosphorylated in an autocrine manner, driving malignant progression.

Ablation of Satb1 in mature CD4 ${ }^{+} T$ cells causes fatal lymphomas with massive $T$ cell skin infiltrates. Our results so far suggested that SATB1 could act as a tumor suppressor in T cells with deregulated NOTCH1 activity. To confirm that Satb1 expression prevents the malignant transformation of truly mature (postthymic) $\mathrm{CD} 4^{+}$ $\mathrm{T}$ cells upon deregulated Notch1 expression, we next generated $C D 4^{\text {Cre }} E R^{T 2} S a t b 1^{f l / f} R o s a 26^{N 1-I C D}$ mice and $C D 4^{C r e} E R^{T 2} R o s a 26^{N 1-I C D}$ littermates. In this system, Cre is selectively activated in mature $\mathrm{CD}^{+} \mathrm{T}$ cells in adult mice (15). Administration of tamoxifen in 6to 8-week-old triple-transgenic mice again led to adenopathy and splenomegaly (Figure $4 \mathrm{~A}$ ), while tamoxifen-injected $C D 4^{C r} E R^{T 2}$ Rosa $26^{\text {N1-ICD }}$ or vehicle-treated and untreated $C D 4^{\mathrm{Cre}} E R^{T 2} S a t b 1^{f / f f}$ Rosa $26^{\text {N1- } I C D}$ mice did not show signs of disease. Examination of the skin of mice with advanced disease showed dermal damage, with obvious $\mathrm{CD}^{+}$lymphocytic infiltrates in the dermis and, to a lesser extent, in the epidermis (Figure 4B), as well as a trend to enlarged spleen and liver (Supplemental Figure 2), albeit less pronounced than in the CD11c-driven model. In CTCL, a disease in which NOTCH1 is overexpressed in a stage-dependent manner (3), homing of T cells to the skin is associated with the upregulation of the chemokine receptors CCR4, CCR6, and CCR10 (16, 17). Supporting a role for SATB1 as a CTCL suppressor, tamoxifendriven ablation of $S a t b 1$ in $\mathrm{CD} 4^{+} \mathrm{T}$ cells (unlike their $\mathrm{CD} 8^{+}$counterparts, as shown in Supplemental Figure 2) resulted in the overexpression of CCR4 in $C D 4^{C r e} E R^{T 2} R o s a 26^{N 1-I C D}$ mice compared with vehicle controls (Figure 4C).

Tamoxifen-induced Satb1 silencing associated with Notch1 overexpression in mature $\mathrm{CD} 4^{+} \mathrm{T}$ lymphocytes produced peripheral cells with convoluted cerebriform nuclei, characteristic of Sézary cells, with the presence of malignant $\mathrm{CD} 3^{+} \mathrm{CD} 4^{+}$lymphocytes (Figure 4D). Interestingly, the majority of these malignant $\mathrm{T}$ cells coexpress CD8 at terminal stages (Figure 4E), along with the myeloid marker CD11b, a determinant also found in $\mathrm{CD}^{+} \mathrm{T}$ cells in an experimental autoimmune encephalomyelitis mouse model (18) (Figure 4E). Mice without the $C D 4 E R^{T 2} C r e$ gene do 
A
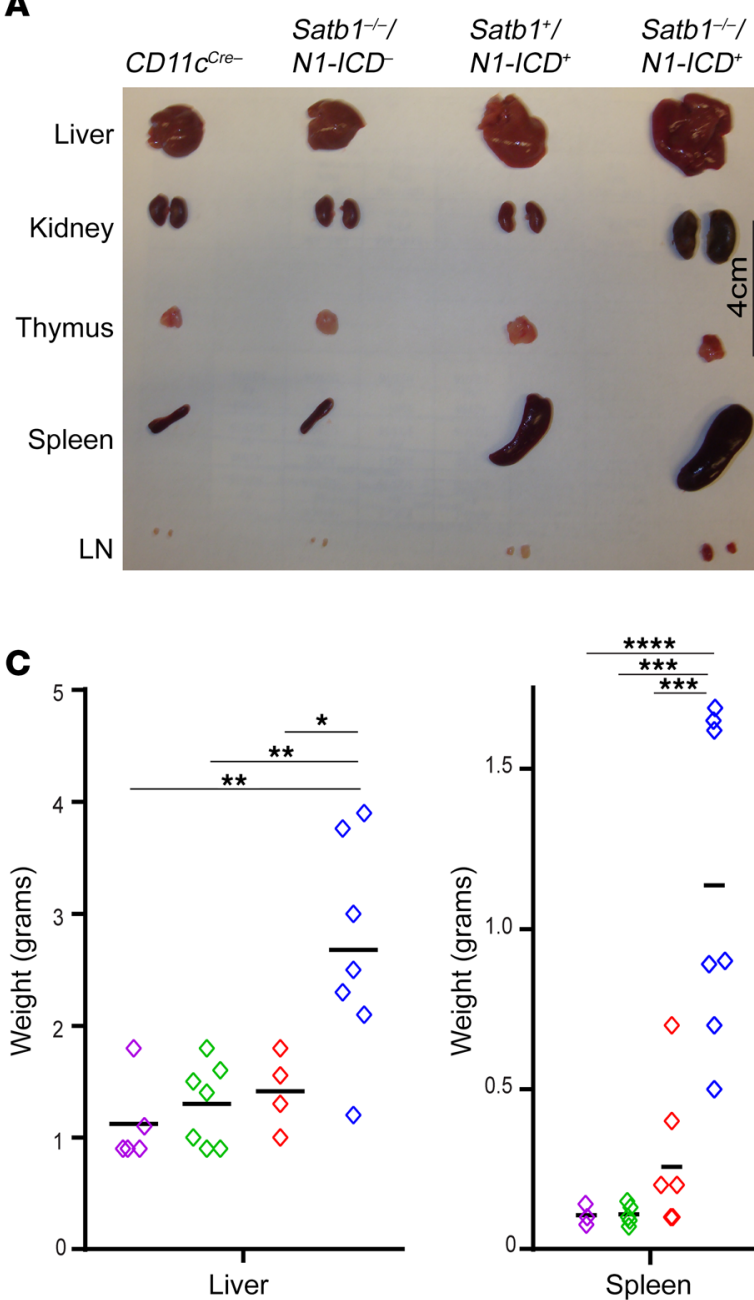

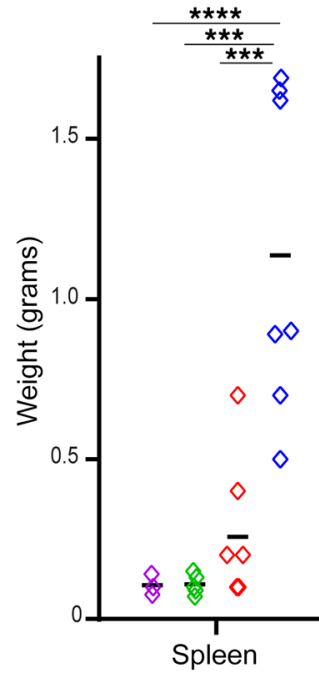

B

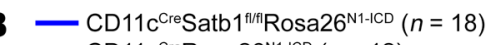

- CD11c CreRosa26 ${ }^{\mathrm{N}-\mathrm{CCD}}(n=12)$

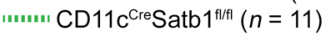

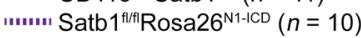

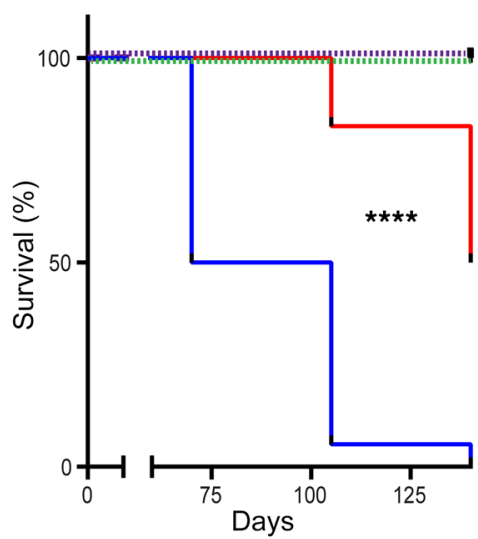

$\diamond$ Satb1 $1^{\mathrm{fl} / \mathrm{f}}$ Rosa26 $6^{\mathrm{N} 1-\mathrm{ICD}}$

$\diamond \mathrm{CD} 11^{\mathrm{C} \text { CreSatb }} 1^{\text {tifil }}$

$\diamond$ CD11C ${ }^{\text {Cre }}$ Rosa26 $6^{\mathrm{N} 1-\mathrm{ICD}}$

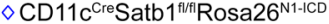

D

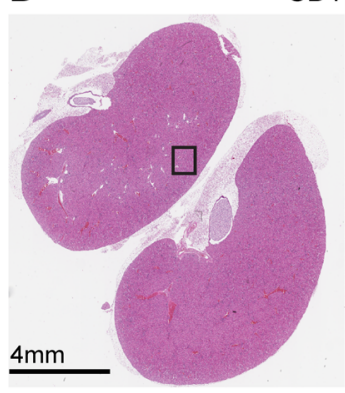

CD11 $\mathrm{C}^{\mathrm{Cre}}$

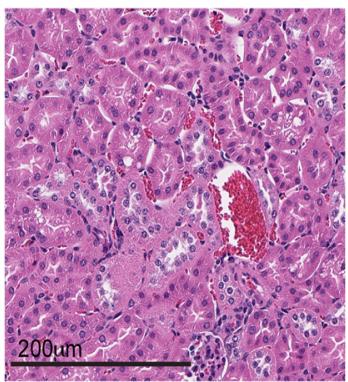

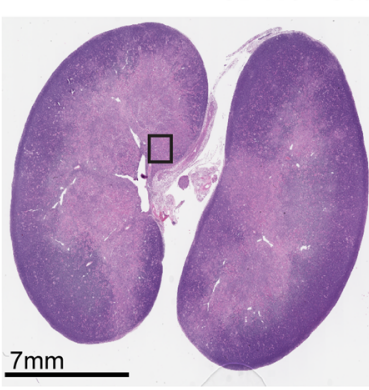
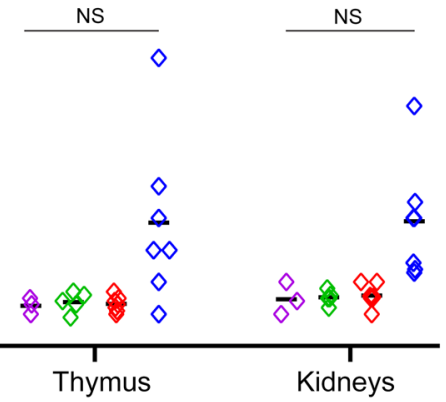

CD11c CreSatb1 1/ffl Rosa26 ${ }^{\text {N1-ICD }}$

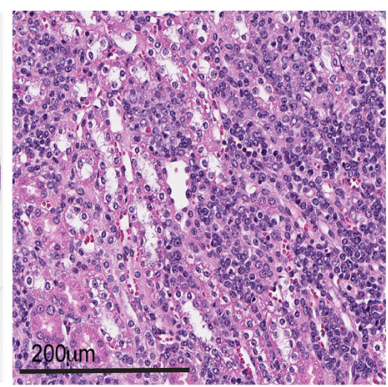

Figure 1. Concurrent ablation of Satb1 and increased expression of Notch1 in mature T cells results in lethal adenopathy. (A) Representative sizes of dif-

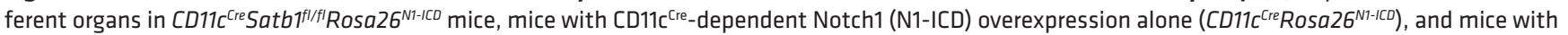

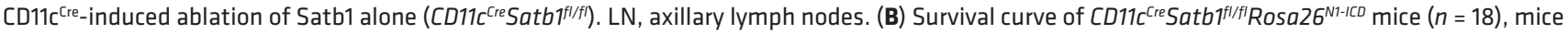

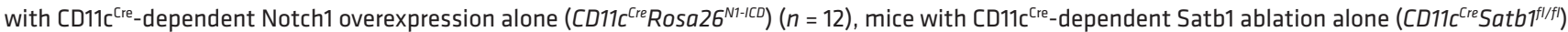
$(n=10)$, and mice without $C D 11 c^{\text {Cre }}(n=11)$. Log-rank (Mantel-Cox) test: ${ }^{* * * *} P<0.0001$. (C) Weight of different organs in CD11c ${ }^{\text {Cre }} S a t b b^{f l / f I} R o s a 26^{N 1-1 C D}$ mice $(n$

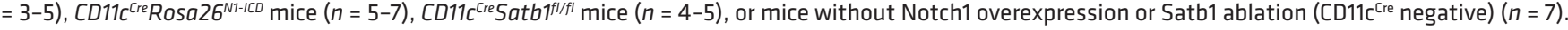
One-way ANOVA with Tukey's multiple-comparison test: ${ }^{*} P<0.05$; ${ }^{*} P \leq 0.01$; ${ }^{* *} P \leq 0.001$; ${ }^{* * *} P \leq 0.0001$. (D) Representative hematoxylin and eosin staining of kidneys of CD11c $c^{\text {Cre }}$-negative mouse (left) versus CD11C $c^{\text {Cre } S a t b 1^{f / f f} / R o s a 26^{N 1-1 C D}}$ mice (right).

not present with splenomegaly, unlike their $C D 4 E R^{T 2} C r e$-positive counterparts. Together, these data indicate that $S a t b 1$ prevents the expression of the chemokine receptors of Notch-activated $\mathrm{CD}^{+}$mature lymphocytes that promote the homing of malignant T cells to the skin.
SATB1 silencing differentiates CTCLs from other peripheral $T$ cell malignancies. To determine whether SATB1 repression plays a role in the pathogenesis of human lymphoproliferative disorders derived from $\mathrm{CD}^{+}$and $\mathrm{CD}^{+} \mathrm{T}$ cells, we first analyzed the expression of SATB1 in malignant $\mathrm{T}$ cells in the bone marrow from 
A

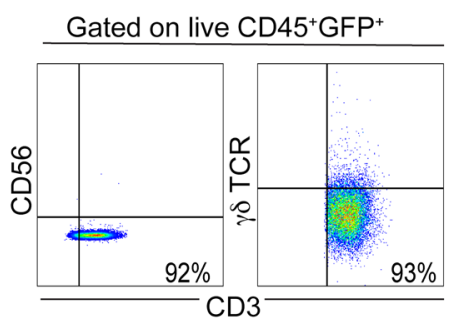

B

CD11 $\mathrm{C}^{\mathrm{Cre}}$ Rosa26 $6^{\mathrm{N} 1-\mathrm{ICD}}$ (gated on live $\mathrm{CD}^{2} 5^{+} \mathrm{CD}^{+} \mathrm{GFP}^{+}$)

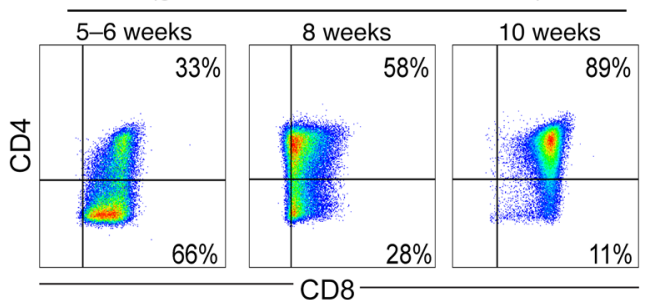

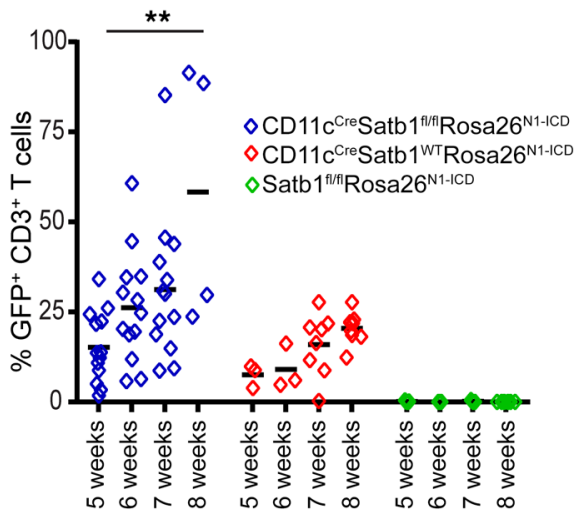

C

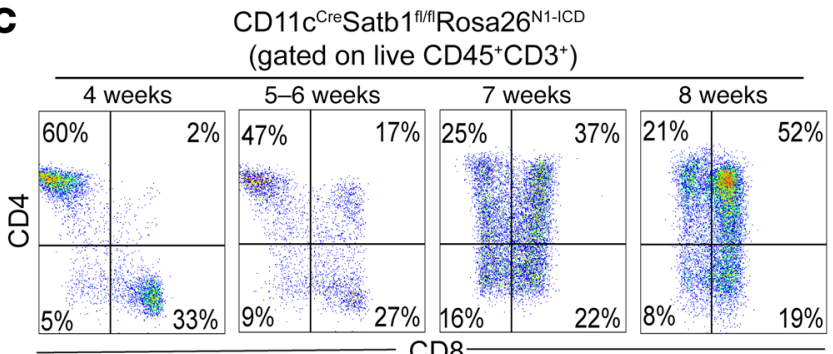

D $\quad$ CD11c Cre Rosa26 $6^{\mathrm{N} 1-\mathrm{ICD}}$ (gated on live $\mathrm{CD} 45^{+} \mathrm{CD} 3^{+}$)

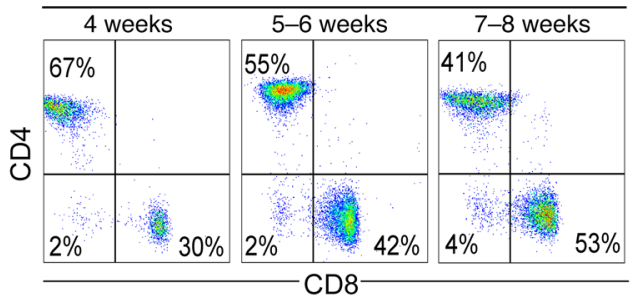

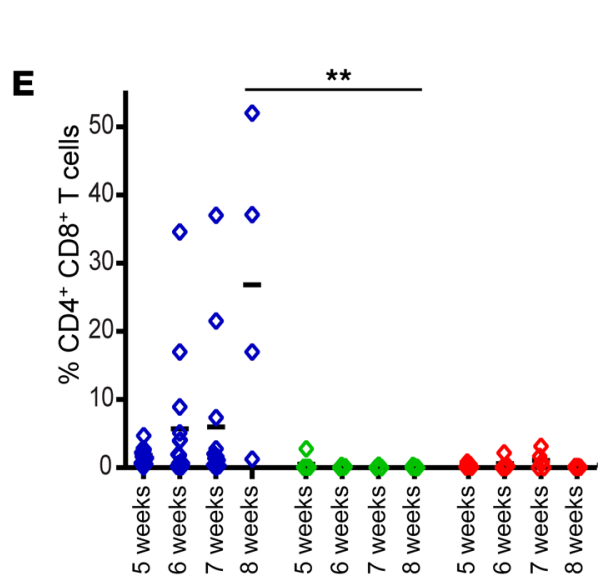
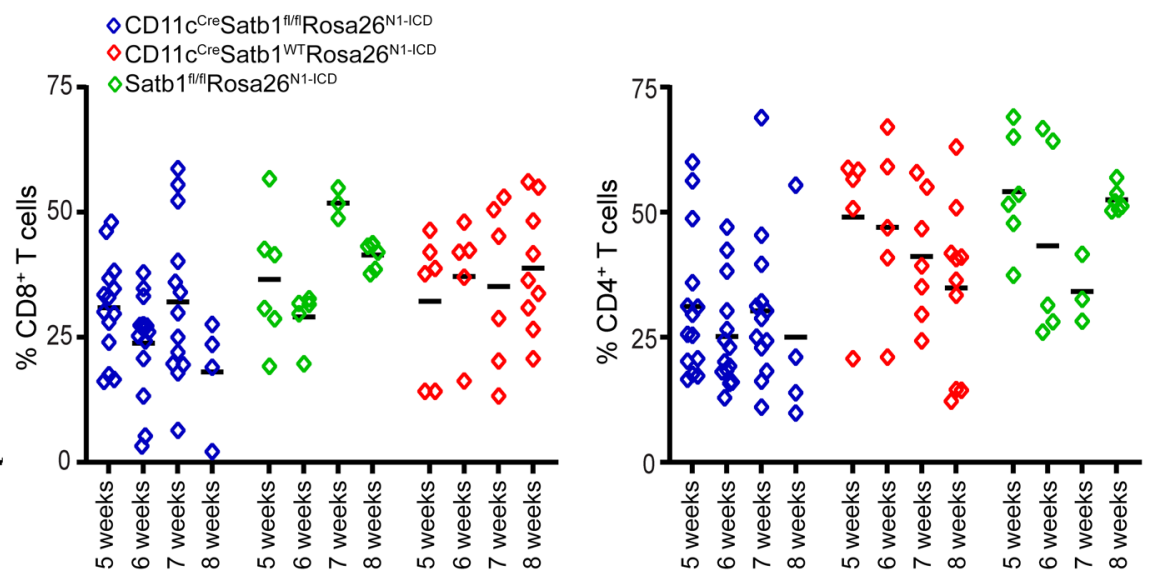

Figure 2. Satb1 ablation transforms the Notch-dependent expansion of CD8 ${ }^{+} T$ cells into a full-blown CD4+CD8 ${ }^{+} T$ cell lymphoma. (A) Notch-overexpress-

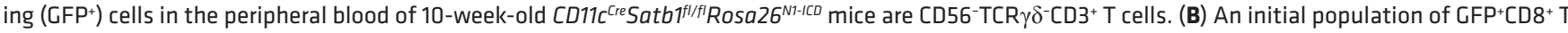
cells overexpressing Notch1 progressively transforms into $C D 4^{+} C D 8^{+} T$ cells at advanced stages of malignant progression, with quantitative representation

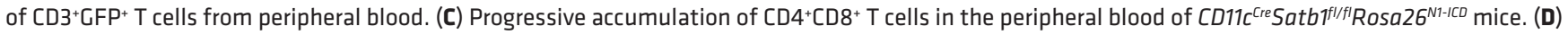
Progressive expansion of $C D 8^{+} T$ cells, but not $C D 4^{+} C D 8^{+}$malignant lymphocytes, in Notch-overexpressing mice without Satb1 ablation. (E) Quantitative

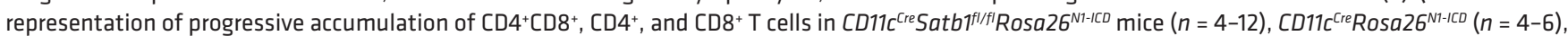
and $C D 11 C^{C r e}$-negative mice $(n=6-9)$. Two-tailed Student's $t$ test $(\mathbf{B}$ and $\mathbf{E}):{ }^{* *} P \leq 0.01$.

a group of 16 patients with a confirmed diagnosis of T cell large granular lymphocyte (T-LGL) leukemia $(n=7)$, T cell prolymphocytic leukemia (T-PLL; $n=6)$, and Sézary syndrome $(n=3)$.

In malignant $\mathrm{T}$ cells from 7 patients with T-LGL leukemia specifically driven by malignant $\mathrm{CD}^{+} \mathrm{CD}^{+}$lymphocytes (Figure $5 \mathrm{~A}$ ), we found that SATB1 was expressed at reproducibly higher levels than in activated $\mathrm{CD} 8^{+} \mathrm{T}$ cells from healthy subjects by intracellular flow cytometric analysis (Figure 5D).

In 4 out of 5 patients with T-PLL driven by malignant CD $4^{+}$ $\mathrm{T}$ cells, SATB1 expression was even higher than in activated $\mathrm{CD}^{+} \mathrm{T}$ cells from healthy donors (Figure 5, A and D). Similarly,
SATB1 was overexpressed in malignant $\mathrm{CD}^{+}{ }^{+} \mathrm{CD} 4^{-} \mathrm{CD}^{-}$cells in one of these patients, as well as in malignant cells from a different T-PLL patients driven by $\mathrm{CD}^{+} \mathrm{CD} 4^{+} \mathrm{CD} 8^{+}$cells (Figure $5, \mathrm{~A}$ and D). In contrast, we found a significant reduction in the levels of SATB1 in malignant $\mathrm{T}$ cells in the bone marrow of 3 different Sézary syndrome patients (Figure 5, B and D). Further supporting the notion that SATB1 repression is a common event in CTCL, low levels of SATB1 were found in $\mathrm{CD}^{+} \mathrm{CD}^{+} \mathrm{CD} 7^{-} \mathrm{T}$ cells sorted from the peripheral blood of 11 additional patients compared with $\mathrm{CD}^{+} \mathrm{T}$ cells from healthy donors (Figure 5, C and D). Therefore, SATB1 silencing appears to be also a pathogenic factor in mycosis 

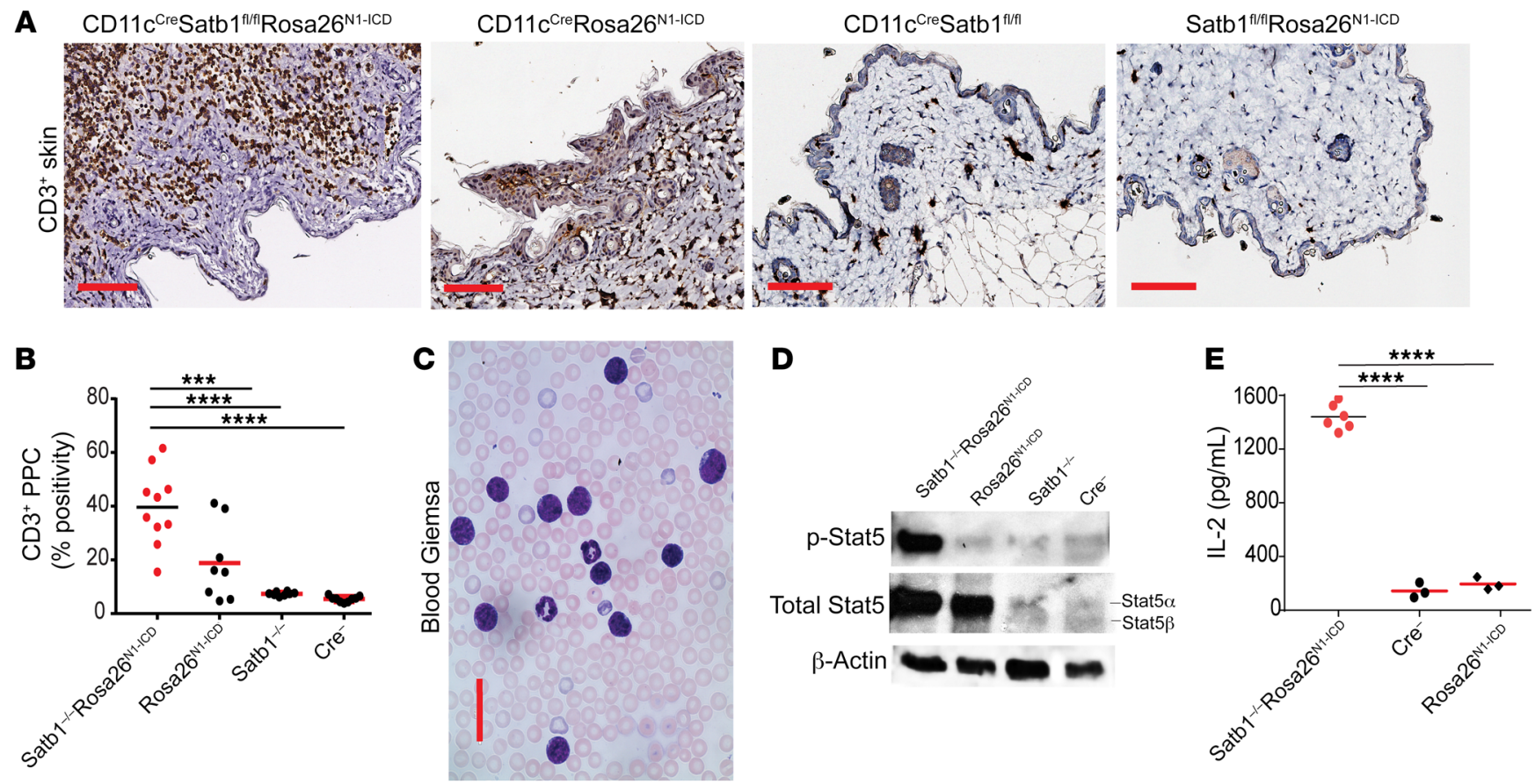

Figure 3. Satb1 ablation and Notch activation cooperate to transform postthymic CD8 ${ }^{+} \mathrm{T}$ lymphocytes into skin-homing lymphoma cells with phosphor-

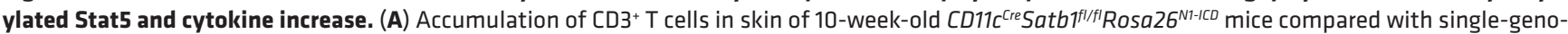

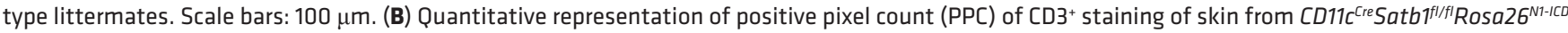

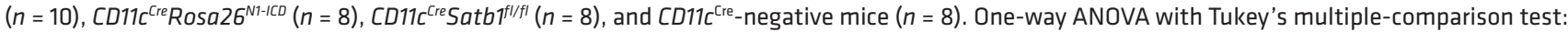

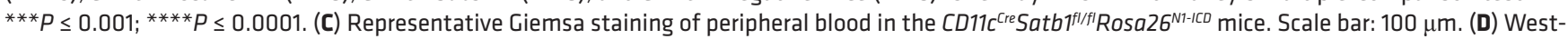
ern blot analysis of protein extracts from GFP+ (Notch1-overexpressing) cells sorted from the bone marrow of $C D 11 c^{\mathrm{Cre}} S a t b f^{f / / f I} R o s a 26^{N-1 C D}$ and $C D 11 c^{C r e}$

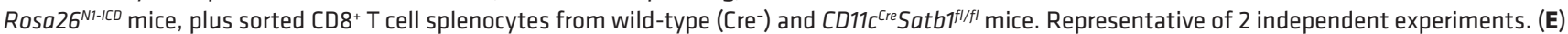
Immunopurified CD3 ${ }^{+}$T cells $\left(2 \times 10^{5}\right)$ were stimulated with $0.5 \mu \mathrm{g} / \mathrm{mL}$ PMA and $1 \mu \mathrm{g} / \mathrm{mL}$ ionomycin in RMP1 with $10^{\circ} \%$ FBS for 4 hours at $37^{\circ} \mathrm{C}$. Superna-

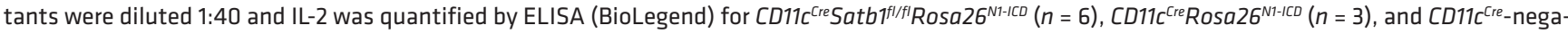
tive mice $(n=3)$. One-way ANOVA with Tukey's multiple-comparison test: ${ }^{* * *} P \leq 0.0001$.

fungoides, although it is not universally found in malignant cells, as in Sézary syndrome. These data validate the relevance of the phenotype identified in our tumor model, and indicate that SATB1 repression is a common pathogenic driver in cutaneous $\mathrm{T}$ cell malignancies (but not other peripheral $\mathrm{T}$ cell malignancies) and acts by dysregulating the expression of chemokine receptors that promote skin homing.

Reversing $\mathrm{H} 3 \mathrm{~K} 9$ trimethylation, but not targeting $\mathrm{H} 3 \mathrm{~K} 27 \mathrm{trimeth}$ ylation, rescues protective SATB1 activity in Sézary $T$ cells. As supported by emerging genomic analysis and recent publications (4), $S A T B 1$ downregulation in Sézary cells is not the result of deletions or point mutations. To determine how SATB1 is silenced in peripheral $\mathrm{T}$ cell malignancies, we focused on the activity of polycomb repressive complex 2 (PRC2) and histone-lysine $N$-methyltransferase suppressor of variegation 3-9 homolog 1 and 2 (SUV39H1/2) in HuT78 Sézary cells, which have been reported to be SATB1 deficient (19). Epigenetic repression often requires coordinated gain of H3K9 trimethylation and H3K27 trimethylation (20) to effectively occlude DNA $(21,22)$. To test this possibility, we quantified occupancy of $\mathrm{H} 3 \mathrm{~K} 9 \mathrm{me}^{3}$ at the SATB1 promoter. We performed chromatin immunoprecipitation sequencing (ChIP-seq) on several leukemia cell lines with various levels of SATB1 expression - HuT78 (low expression), Jurkat (high expression), and RAJI (no detectable expression) - to ascertain the regulatory landscape near the SATB1 transcription start site (TSS) (GSE 159962) (Supplemental Table 1). Immunoprecipitations were performed on all cell lines for H3K27 $\mathrm{me}^{3}$, H3K9 $\mathrm{me}^{3}$, and H3K27ac to reveal several sites of enrichment as shown in Supplemental Figure 3. The analysis revealed no occupancy of the marker H3K27 $\mathrm{me}^{3}$ for Jurkat cells, no occupancy of H3K27ac for K562 cells, and no occupancy for any markers for RAJI cells near the TSS of SATB1. Ensembl regulatory regions at approximately $4.8 \mathrm{~kb}$ and approximately $5.6 \mathrm{~kb}$ from the TSS of SATB1 were also found for repressive histone markers $\mathrm{H} 3 \mathrm{~K} 27 \mathrm{me}^{3}$ and $\mathrm{H} 3 \mathrm{~K} 9 \mathrm{me}^{3}$, respectively. We found several regions in the H3K9me ${ }^{3}$ pull-down of ChIP-seq from which we designed primers to validate by ChIP-PCR using specific antibodies or an irrelevant IgG isotype in control immunoprecipitations, as shown in Figure 6A. Enrichment of $\mathrm{H} 3 \mathrm{~K} 27 \mathrm{me}^{3}$ and $\mathrm{H} 3 \mathrm{~K} 9 \mathrm{me}^{3}$ appeared in HuT78 cells in the approximately 4.8 -kb and approximately 5.6$\mathrm{kb}$ regions, but no significant enrichment was found for a control adjacent sequence (Figure 6B). Out of the 3 regions identified via ChIP-seq for $\mathrm{H} 3 \mathrm{~K} 9 \mathrm{me}^{3}$, region 1 was shown to be enriched in HuT78 cells and RAJI cells in which SATB1 is repressed, but not in Jurkat cells in which SATB1 is highly expressed (Figure 6C).

To define the requirement of PRC2-dependent SATB1 repression for the expansion of Sézary T cells, we next treated HuT78 cells with increasing concentrations of the EZH2 inhibitor GSK126. As shown in Figure 6D, inhibition of EZH2 decreased the number of 


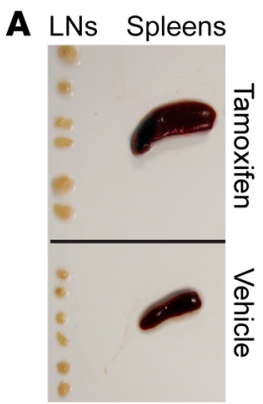

B

E

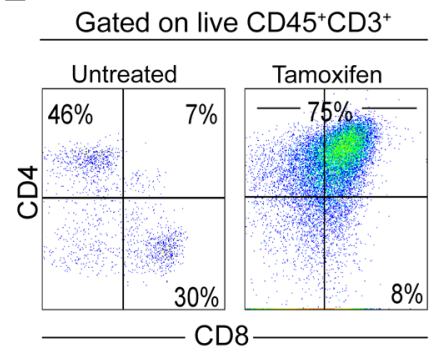

$8 \%$

CD8

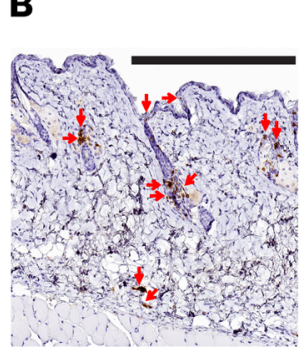

C

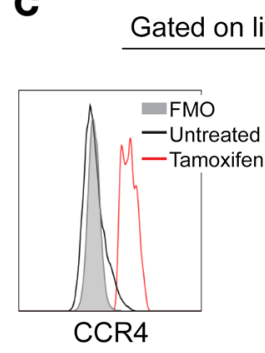

D CD4ER ${ }^{\mathrm{T} 2} \mathrm{Cre}$ model

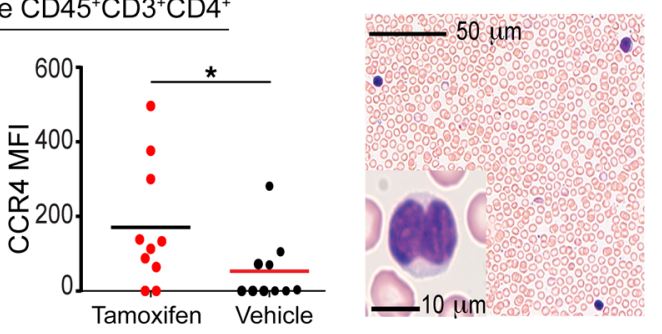

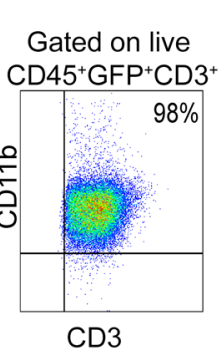

CD3
$\mathbf{F}$

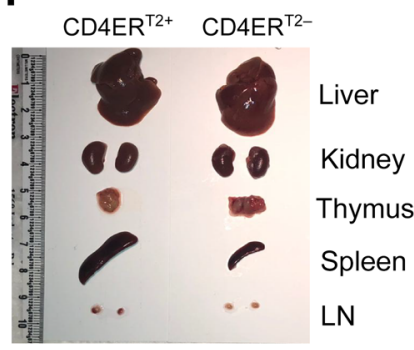

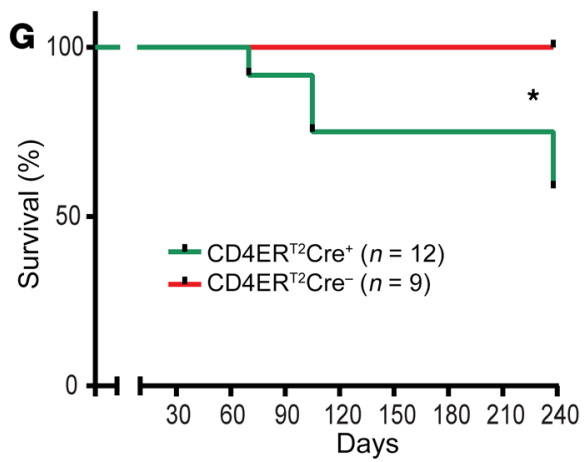

Figure 4. Satb1 ablation and Notch activation cooperate to transform postthymic CD4+ $T$ lymphocytes into skin-homing lymphoma cells. (A) Splenomegaly and adenopathy in a $C D 4^{\mathrm{Cr} e} E R^{T 2} S a t b 7^{f / / f} R o s a 26^{N-1 C D}$ mouse 2 months after tamoxifen-mediated activation of transgenes. Representative differences in the size of lymph nodes (LNs) and spleens from different $C D 4^{C r e} E R^{T 2} S a t b 7^{f l / f I} R o s a 26^{N 1-1 C D}$ mice injected with tamoxifen $(n=9)$ versus corn oil (vehicle control) $(n=9)$. (B) Accumulation of CD3 ${ }^{+}$T cells (red arrows) in the skin of $C D 4^{C r e} E R^{T 2} S a t b 7^{f / f f} R o s a 26^{N 1-1 C D}$ mice, 10 weeks after tamoxifen administration. Scale bar: $300 \mu \mathrm{m}$. (C) Representative histogram analysis (left) and median fluorescence intensity (MFI, right) of the expression of CCR4 in CD3+CD4+ $T$ cells in the peripheral blood of $C D 4^{C r e} E R^{T 2} S a t b 1^{f l / f I} R o s a 26^{N 1-1 C D}$ mice, treated with tamoxifen $(n=10)$ versus corn oil $(n=10)$. Two-tailed Student's $t$ test: ${ }^{*} P<$ 0.05. (D) Representative Giemsa staining of peripheral blood in the same mice. Scale bar: $50 \mu \mathrm{m}$. A detail of a cerebriform cell is also shown. Scale bar: 10 $\mu \mathrm{m}$. (E) Expansion of CD4 ${ }^{+} C D 8^{+} C D 11 b^{+} T$ cells in the peripheral blood of $C D 4^{C r e} E R^{T 2} S a t b 7^{f / / f l} R o s a 26^{N-1 C D}$ mice 10 weeks after tamoxifen challenge. (F) Splenomegaly and adenopathy in a $C D 4^{\mathrm{Cr} e} E R^{T 2} S a t b 7^{f / / f 1} R o s a 26^{N-1 C D}$ mouse 2 months after tamoxifen-mediated activation of transgenes. Representative differences in the size of $L N s$ and spleens from different $C D 4^{\text {Cre }} E R^{T 2} S a t b f^{f / / f I} R o s a 26^{N-1 C D}$ versus $C D 4^{C r e} E R^{T 2}$-negative mice. (C) Survival curve of $C D 4^{\text {Cre } E} R^{T 2} S a t b 7^{f l / f l}$ Rosa26 $6^{N 1-C D}$ mice $(n=12)$ versus mice without $C D 4^{C r e} E R^{T 2}(n=9)$. Log-rank (Mantel-Cox) test: ${ }^{*} P<0.05$.

viable Sézary cells and increased SATB1 mRNA expression (Figure $6 \mathrm{~K})$. Treatment with $10 \mu \mathrm{M}$ GSK126 decreased the occupancy of $\mathrm{H} 3 \mathrm{~K} 27 \mathrm{me}^{3}$ in the approximately 4.8 -kb site of the SATB1 promoter compared with the vehicle control (DMSO) (Figure 6H).

SUV39H1/2 enzymes are primarily responsible for eliciting and maintaining H3K9 trimethylation at constitutive heterochromatin regions $(23,24)$. Accordingly, the histone lysine methyltransferase inhibitor chaetocin reduced the number of viable Sézary cells (Figure 6E). These effects were not the result of the nonselective activity of chaetocin, as F5446, a recently reported SUV39H1/2-specific inhibitor (25), also blocked Sézary cell expansion at 50\% inhibitory concentration $\left(\mathrm{IC}_{50}\right)$ values in the nanomolar range (Figure 6F), which are 4 -fold lower than those needed to restore effector gene expression in tumor-reactive $\mathrm{T}$ cells (25). In contrast, the $\mathrm{IC}_{50}$ of romidepsin (Figure $6 \mathrm{G}$ ) was 44 -fold higher than that of chaetocin (Figure 6E). Interestingly, combinations of the HDAC inhibitor romidepsin and SUV39H1/2 inhibitor chaetocin abrogated the protective effect of either individual treatment. Treatment of HuT78 cells at 72 hours resulted in significantly increased SATB1 mRNA expression (Figure 6L). Treatment of HuT78 cells with chaetocin, F5446, or romidepsin versus the DMSO control showed that F5446 and romidepsin produce a cytostatic effect compared with chaetocin (Figure 6M). RNA-seq of HuT78 cells collected after 72 hours of treatment with chaetocin and F5446 revealed pathways associated with negative regulation of $\alpha \beta$ T cell activation, methyltransferase activity, and chemokine binding pathways, while having positive regulation of cellular senescence, nucleosome binding, and histone modification (GSE 159963) (Supplemental Figure 4 and Supplemental Tables 2 and 3). Together, these data suggest that H3K9 trimethylation, H3K27 deacetylation, and H3K27 trimethylation are potential targetable mechanism to rescue SATB1 expression and abrogate malignant cell growth.

SUV39H1/2 inhibition derepresses SATB1 and arrests primary Sézary cell expansion more effectively than romidepsin. To compare the effects of methyltransferase, histone deacetylase, and EZH2 inhibitors against CTCL, we next treated purified malignant primary $\mathrm{CD}^{+} \mathrm{CD} 4^{+} \mathrm{CD} 26^{-}$cells from the apheresis of 4 Sézary patients with high blood tumor burden ( $>1,000$ malignant cells/ $\mu \mathrm{L}$ ) (Figure 7, A and B) as well as 4 additional Sézary patients with $\mathrm{CD}^{+} \mathrm{CD}^{+}$cells from peripheral blood (Supplemental Figure 5A). Notably, both methyltransferase inhibitors chaetocin and F5446 were effective at abrogating the expansion of Sézary cells from all patients, at $\mathrm{IC}_{50}$ values in the nanomolar range. In contrast, the class I HDAC inhibitor romidepsin, FDA approved for the treatment of relapsed/refractory CTCL, only decreased the number of viable Sézary cells from the same patients at doses more than 
A $\quad T-L G L$

Gated on $\mathrm{CD}^{+} \mathrm{CD} 4-\mathrm{CD}^{+}$
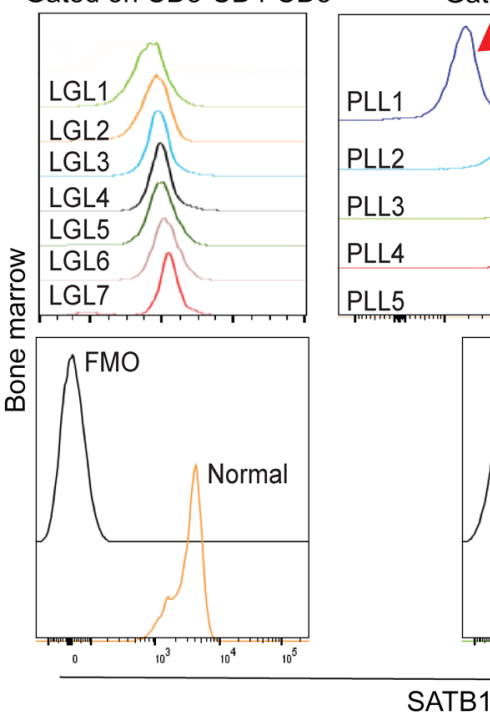

\section{D}

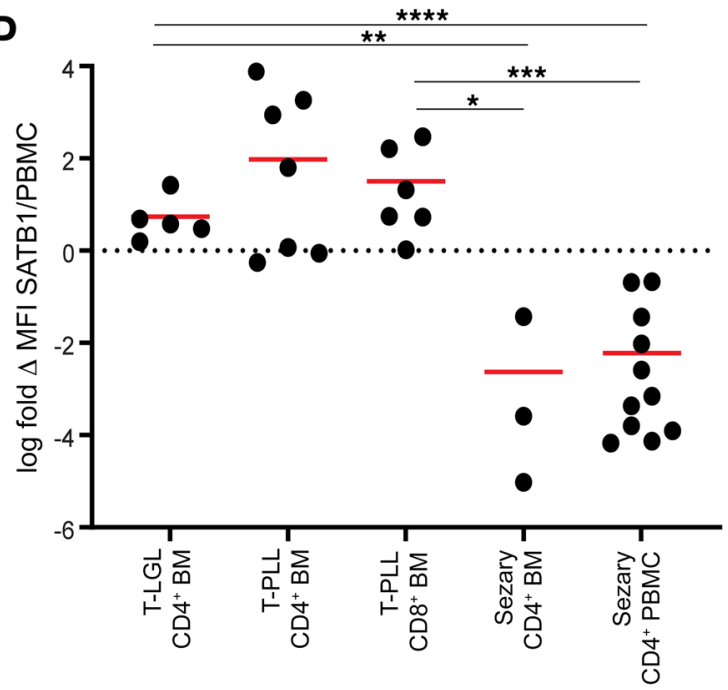

8-fold higher than F5446, under identical conditions, while treatment with the EZH2 inhibitor GSK126 was not effective under any condition (Figure 7, A and B). Primary Sézary cells treated with F5446, as opposed to chaetocin, significantly increased the expression of SATB1 mRNA (Figure 7C). Accordingly, ChIP-PCR analysis of purified primary Sézary cells showed negligible H3K27me ${ }^{3}$ occupancy, compared with $\mathrm{H} 3 \mathrm{~K} 9 \mathrm{me}^{3}$ in their respective regions of occupancy in the SATB1 promoter (Figure 7, D and E, and Supplemental Figure 5, B and C). In order to determine the effects of $S A T B 1$ recovery in primary Sézary samples, malignant $C D 4^{+} \mathrm{CD} 26$ cells were retrovirally transduced to ectopically express SATB1. Levels of p-STAT5 were assessed in positively transduced SATB1high cells and compared to cells expressing endogenous levels of $S A T B 1$ from the same donor where an increase in p-STAT5 upon $S A T B 1$ recovery was observed (Figure $7 F$ ). The recovery of SATB1 resulted in an impairment of proliferative potential (Figure $7 G$ ) and is congruent with our observation with HuT78 cells where $S A T B 1$ recovery from epigenetic inhibitors in Figure 6 does not
B

Sezary

Gated on $\mathrm{CD}^{+}{ }^{+} \mathrm{CD} 4{ }^{+} \mathrm{CD} 7-$

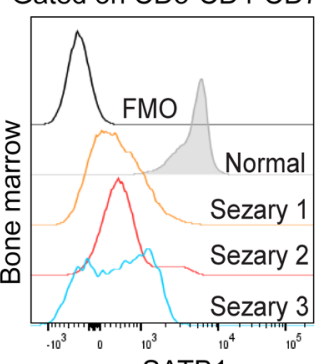

SATB1
C Sezary

Gated on $\mathrm{CD}^{+}{ }^{+} \mathrm{CD} 4^{+} \mathrm{CD} 7^{-}$

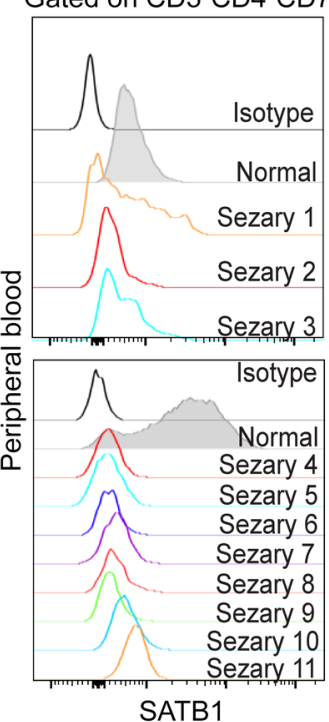

Figure 5. Sézary cells, but not other malignant lymphocytes, consistently exhibit SATB1 downregulation. (A) Expression of SATB1 in the indicated populations of malignant cells in the bone marrow of patients with T-LGL $(n=7)$ or T-PLL $(n=6)$, compared with SATB1 expression in CD8 ${ }^{+}(\mathrm{T}-\mathrm{LGL})$ or $\mathrm{CD} 44^{+}$(T-PLL) T cells activated with anti-CD3/anti-CD28 beads for 48 hours. Gated on live $\mathrm{CD} 45^{+} \mathrm{CD} 3^{+} \mathrm{CD} 4^{+}$or $\mathrm{CD} 8^{+} \mathrm{T}$ cells. FMO, fluorescence minus one. (B) SATB1 downregulation in bone marrow Sézary cells $(n=3)$. (C) SATB1 downregulation in nonactivated peripheral blood Sézary cells ( $n$ $=11$ ) compared with normal donor CD4 ${ }^{+} T$ cells. (D) Log-transformed foldchange values of SATB1 MFI levels of malignant cells over normal CD4 ${ }^{+}$or CD8 ${ }^{+}$PBMCs for T-LGL CD4 ${ }^{+}$bone marrow $(B M)(n=5)$, T-PLL CD4 $4^{+}$BM $(n=$ 7), T-PLL CD8 ${ }^{+} B M(n=6)$, Sézary CD4 $4^{+} B M(n=3)$, and Sézary CD4+ PBMCs $(n=11)$. Two-tailed Student's $t$ test: ${ }^{*} P<0.05 ;{ }^{* *} P \leq 0.01 ;{ }^{* *} P \leq 0.001$; ${ }^{* * * *} P \leq 0.0001$.

induce significant apoptosis. Therefore, SUV39H1/2-mediated repression of SATB1 emerges as a major potential therapeutic target to restore tumor-suppressor SATB1 expression in Sézary patients in a manner that preserves healthy cells.

\section{Discussion}

Here we show that SATB1 silencing in PTCL cells cooperates with NOTCH1 activation to drive mature $\mathrm{T}$ cell expansion through STAT5 activation and CCR 4 expression. Correspondingly, we found that SATB1 is epigenetically repressed in human Sézary syndrome through $\mathrm{H} 3 \mathrm{~K} 27 \mathrm{me}^{3}$ - and $\mathrm{H} 3 \mathrm{~K} 9 \mathrm{me}^{3}$-mediated chromatin occlusion, and SUV39H1/2 inhibition has significant antiproliferative effects in malignant cells from multiple Sézary syndrome patients.

CTCL is a disease with no known curative therapies that is accompanied by painful and pruritic skin lesions causing both disfigurement and significant deterioration in quality of life. At advanced stages, the disease progresses rapidly with short survival. With the exception of palliative anti-CCR4 antibodies to 
A

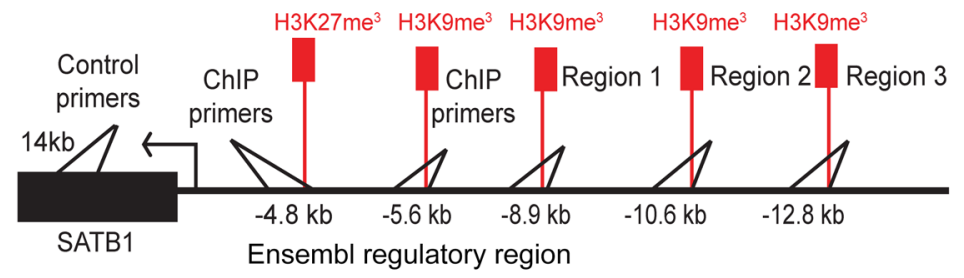

B
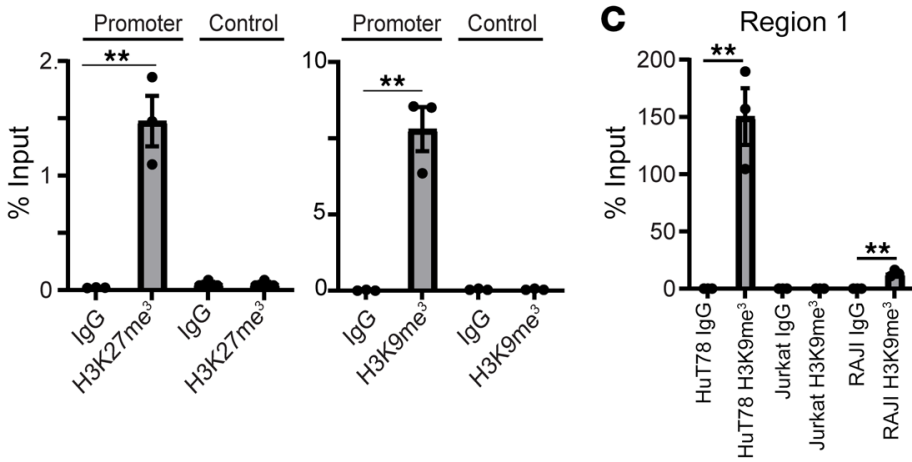

Region 2 Region 3

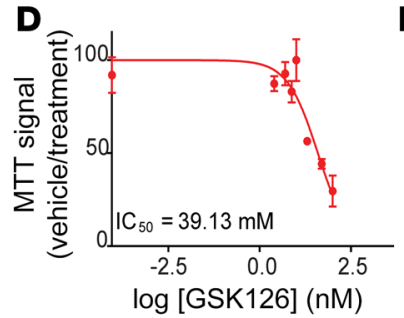

\section{$\mathbf{E}$}

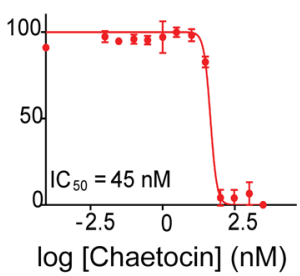

$\mathbf{F}$

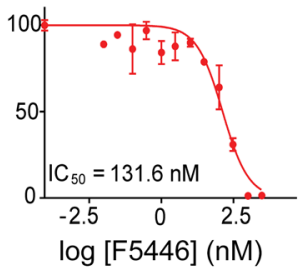

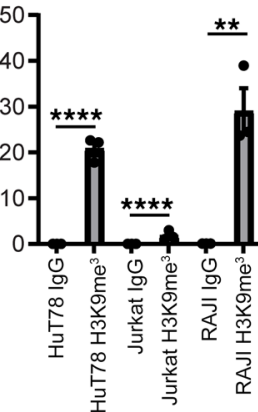

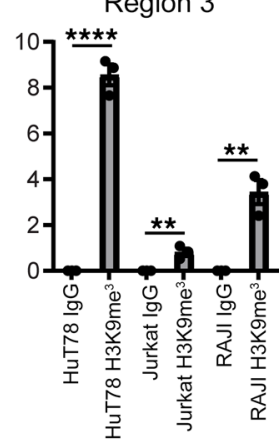

G

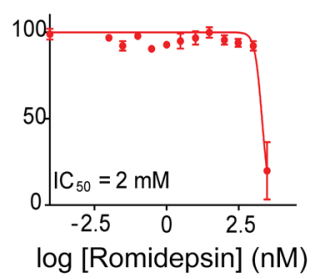

H

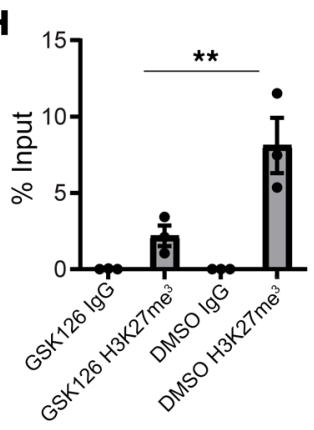

$\mathbf{K}$

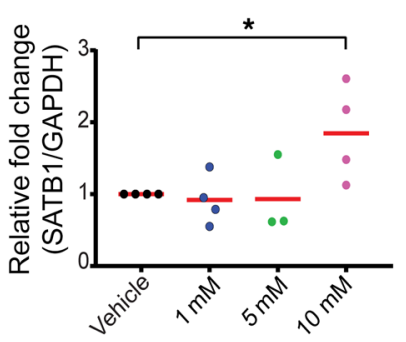

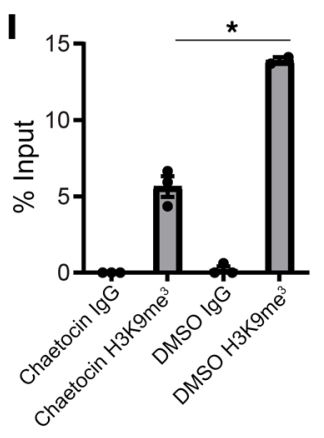

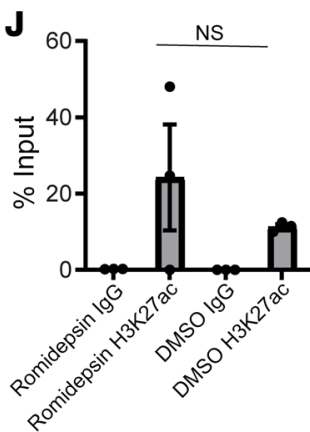

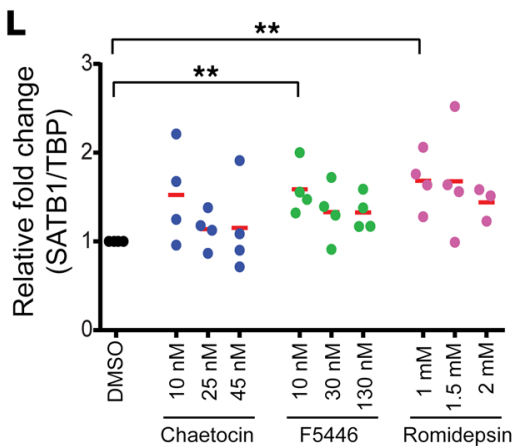

M

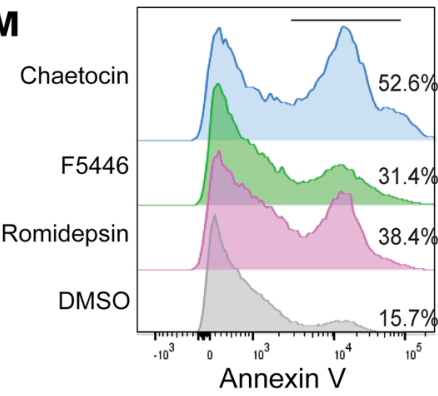


Figure 6. H3K27me and $\mathrm{H} 3 \mathrm{~K} 9 \mathrm{me}^{3}$ occlude the SATB1 promoter in a

Sézary cell line. (A) Schematic depiction of primer sites for the regulatory and control regions for ChIP-PCR analysis in B and C. (B) ChIP quantified by real-time q-PCR with anti-H3K27me (clone C36B11) (left) or anti-H3K9me ${ }^{3}$ (Abcam, ab8898) (right) versus the control region (approximately $14 \mathrm{~kb}$ from promoter) and control IgC pull-downs from HuT78 Sézary cells calculated against $2.5 \%$ input values. Regions amplified at predicted occupied region approximately $4.8 \mathrm{~kb}$ for $\mathrm{H}_{3} \mathrm{~K} 27 \mathrm{me}^{3}$ and approximately $5.6 \mathrm{~kb}$ for $\mathrm{H} 3 \mathrm{~K} 9 \mathrm{me}^{3}$ from SATB1 promoter. Representative of 2 independent experiments. (C) ChIP quantified by real-time qPCR with anti-H3K9me ${ }^{3}$ (ab8898) and control IgC pull-downs from HuT78, Jurkat, and RAJl cells lines calculated against $2.5 \%$ input values. Regions amplified based on ChIP-seq data for H3K9me occupied regions near the SATB1 promoter. (D-G) HuT78 cells were treated with vehicle or increasing concentrations of the EZH2 inhibitor GSK126 for 48 hours in duplicate for MTT assay (D), SUV39H1 inhibitors chaetocin (E) and F5446 (F), or the HDAC inhibitor romidepsin (C) in duplicate, and MTT assays were performed after 72 hours. Representative of 2 independent experiments. (H-J) ChIP-PCR experiments on HuT78 cells treated with $\mathrm{IC}_{50}$ values of GSK126 (H), chaetocin (I), and romidepsin (J) pulled down with anti-H3K27 $\mathrm{me}^{3}$ (clone C36B11), antiH3K9me ${ }^{3}$ (Abcam, ab8898), or anti-H3K27ac (Abcam, ab4729), respectively. Representative of 2 independent experiments. (K) RNA was extracted from HuT78 cells identically treated for 48 hours was reversed transcribed, and SATB1 mRNA expression was quantified by q-PCR normalized to human GAPDH mRNA. Data pooled from 4 independent experiments are shown. (L) q-PCR quantification of SATB1 mRNA expression normalized to TATA-binding protein (TBP) mRNA 72 hours after treatment with the indicated doses of chaetocin, F5446, or romidepsin. Pooled from 4 independent experiments. (M) Histogram of annexin V staining on HuT78 cells treated with the $\mathrm{IC}_{50}$ value of chaetocin, $\mathrm{F} 5446$, or romidepsin. Two-tailed Student's $t$ test (B, C, and $\mathbf{H}-\mathbf{L}):{ }^{*} P<0.05 ;{ }^{* *} P \leq 0.01 ;{ }^{* * *} P \leq 0.0001$.

reduce circulating cells in the blood and skin homing (26), no new treatments have been FDA approved for advanced refractory CTCL since romidepsin in 2009. This is due, in part, to a poor understanding of its pathophysiology. We find that expression levels of the master epigenetic organizer SATB1 are significantly reduced in CTCL CD4 ${ }^{+} \mathrm{T}$ cells in the bone marrow and the periphery of multiple Sézary syndrome patients, compared with $\mathrm{CD} 4^{+}$lymphocytes from healthy donors or other peripheral $\mathrm{T}$ cell malignancies. SATB1 is a key regulator of T cell development and maturation, but also governs the function of other mature immune cells $(6,7,27,28)$. Although recent reports suggested that SATB1 could be universally downregulated in CTCL (10), the causes and effects of $S A T B 1$ silencing remain elusive. Using a transgenic model, we found that ablation of Satb1 in mature T cells promotes the malignant and skin-homing properties of mature Notch-activated lymphocytes through a mechanism that involves derepression of CCR4 and constitutive activation of Stat5 signaling. Importantly, NOTCH and STAT5 signaling is activated in a stage-dependent manner in CTCL $(3,29)$, while the homing of malignant T cells to the skin is driven by the upregulation of chemokine receptors such as CCR4 $(16,17)$. Interestingly, phosphorylation of Stat5 requires cooperation between Satb1 ablation and Notch signaling, because the individual events were insufficient to activate Stat5 signaling. Future studies will determine whether this is the result of enhanced secretion of IL-2 in malignant cells or the effect of deregulation in JAK activity.

SATB1 silencing does not appear to be restricted to Sézary syndrome because a recent study identified that among 57 myco- sis fungoides patients, $35 \%$ were deficient in SATB1 or presented low expression specifically in the skin, which was associated with worse outcome (30). Importantly, seminal publications support the notion that SATB1 silencing in Sézary cells is not the result of mutations (4), indicating epigenetic repression. We found that the SATB1 promoter in primary Sézary cells is marked by trimethylated H3K27 and H3K9 and acetylated H3K27. Histone deacetylase inhibitors and SUV39H1/2 partially restored SATB1 and had significant antiproliferative effects on malignant cells. Although H3K27me $\mathrm{m}^{3}$ occupancy is consistently associated with gene repression, transcription factors can still access $\mathrm{H} 3 \mathrm{~K} 27 \mathrm{me}^{3-}$ marked promoters, unlike $\mathrm{H} 3 \mathrm{~K} \mathrm{me}^{3}$-marked chromatin, which remains completely occluded (21). Hence, inhibition of the enzymes primarily responsible for causing and maintaining $\mathrm{H} 3 \mathrm{~K} 9$ trimethylation at constitutive heterochromatin $(23,24)$ (namely, SUV39H1/2) emerges as what could be a novel intervention for urgent testing against refractory CTCL. Our results therefore offer potentially new insight into the pathophysiology of CTCL as well as a mechanistic rationale for targeting histone methyltransferases to abrogate malignant expansion and skin homing in advanced-CTCL patients.

\section{Methods}

Animals and human samples. Genetically Satb1-deficient mice were generated in the Wistar Institute's transgenic facility on a C57BL/6 background $(6,7)$. Rosa26 ${ }^{\mathrm{N}-\mathrm{CCD}}$ mice were provided by Ben Stanger (University of Pennsylvania) and backcrossed for at least 10 generations to a C57BL/6 background. These transgenic mice have a floxed version of Notch1 interrupted by a STOP codon at the permissive locus Rosa26. Satb1 ${ }^{\text {f/f }}$ mice were crossed with CD11 ${ }^{\mathrm{Cre}}$ mice (Taconic, $4196 \mathrm{M})$ and Rosa $26^{\mathrm{N}-\mathrm{ICD}}$ mice to generate triple-transgenic (6) and

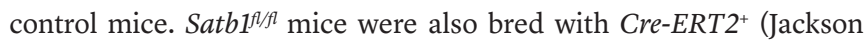

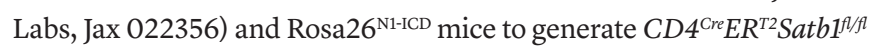
Rosa26 $6^{N-I C D}$ mice. $C D 4^{C r e} E R^{T 2} S a t b 1^{R / A} / R o s a 26^{N-I C D}$ mice were male and injected at 6-8 weeks of age with $75 \mathrm{mg} / \mathrm{kg}$ tamoxifen (MilliporeSigma, T5648) in corn oil (MilliporeSigma, C8267). All animals were maintained in pathogen-free barrier facilities.

Deidentified human bone marrow aspirates were procured under a protocol approved by the H. Lee Moffitt Scientific Review Committee. Sorted bone marrow was maintained in RPMI media with $10 \%$ human serum (MilliporeSigma, H5667), penicillin/streptomycin (Lonza, 17-602E), $100 \mathrm{U} / \mathrm{mL}$ IL-2 (Peprotech, 200-02), and $5 \mathrm{ng} / \mathrm{mL} \mathrm{IL-7}$ (Peprotech, 200-07). $\mathrm{CD} 4^{+} \mathrm{CD} 7^{+} \mathrm{CD} 26^{+}$and $\mathrm{CD}^{+}{ }^{+} \mathrm{CD} 7{ }^{-} \mathrm{CD} 26^{-}$cells were isolated with a Human $\mathrm{CD} 4^{+} \mathrm{T}$ cell Isolation Kit (STEMCELL Technologies, 17952) and Release Human PE Positive Selection Kit (STEMCELL Technologies, 17654) from peripheral blood of patients with Sézary syndrome and cultured in complete RPMI (10\% heatinactivated FBS, $0.5 \mathrm{mM}$ sodium pyruvate [Gibco, 11360070], $2 \mathrm{mM}$ L-glutamine [MilliporeSigma, G7513], $100 \mathrm{IU} / \mathrm{mL}$ penicillin, $100 \mu \mathrm{g} /$ $\mathrm{mL}$ streptomycin [Lonza, 17-602E], and $100 \mathrm{U} / \mathrm{mL}$ IL-2 [Peprotech, 200-02]) in an incubator $\left(37^{\circ} \mathrm{C}, 5 \% \mathrm{CO}_{2}\right)$. Memory $\mathrm{CD}^{+} \mathrm{T}$ cells from peripheral blood of normal donors were isolated using a Human Memory CD4 ${ }^{+} \mathrm{T}$ Cell Enrichment Kit (STEMCELL Technologies, 19157) and cultured in exactly the same way as peripheral blood Sézary cells.

Retroviral transduction. Primary Sézary $\mathrm{CD}^{+} \mathrm{CD} 26^{-}$cells were activated with anti-human CD3/anti-human CD28 Dynabeads (Gibco, 1132D) and transduced with retrovirus produced using Phoenix- 
A
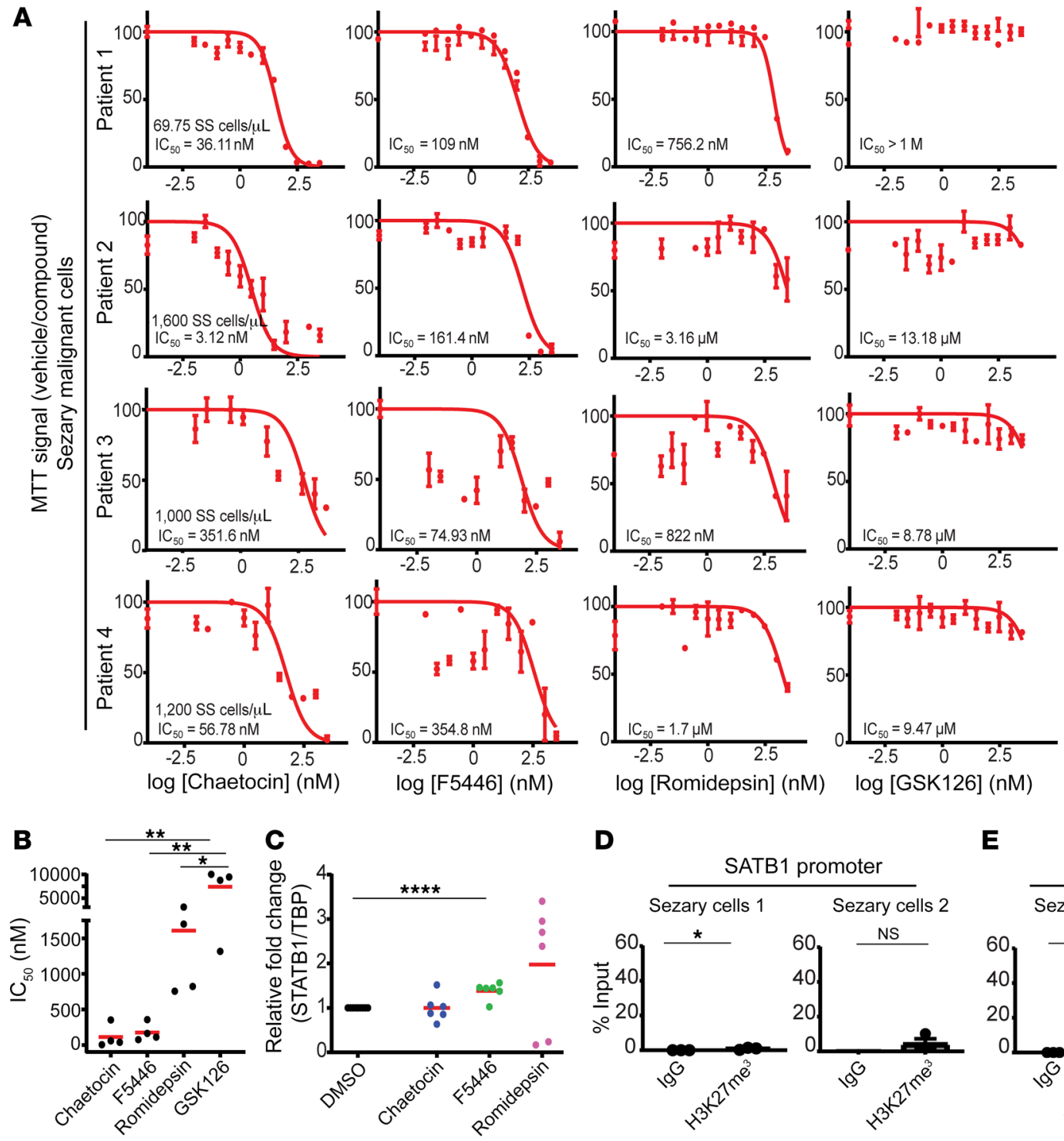

D

E
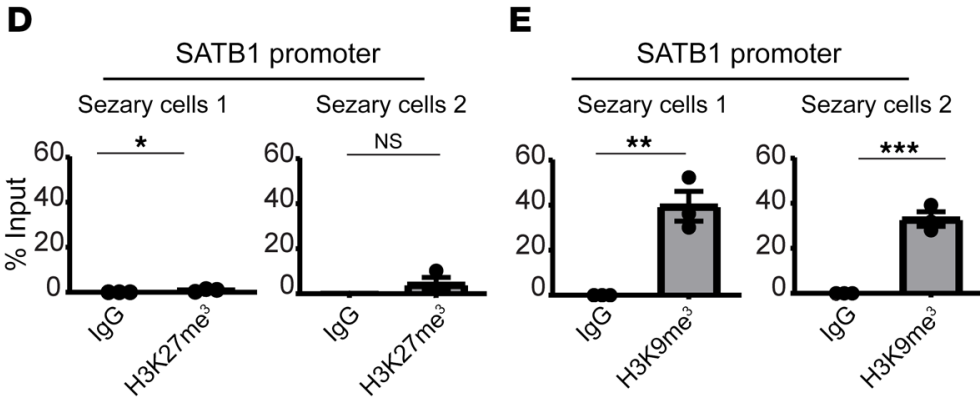

$\mathbf{F}$

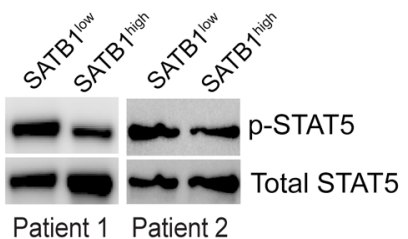

G

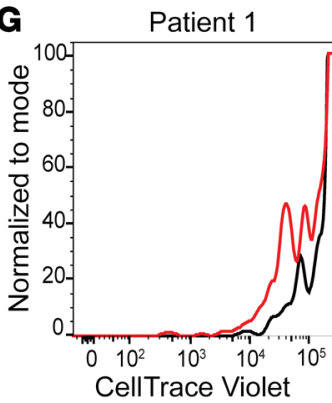

Patient 2

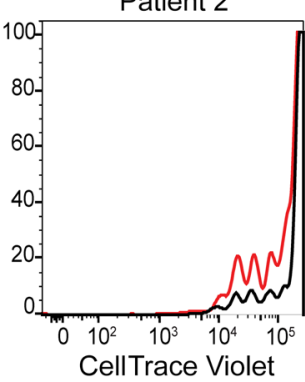

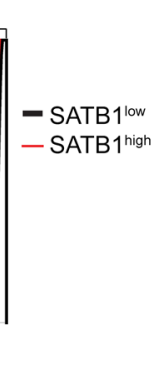

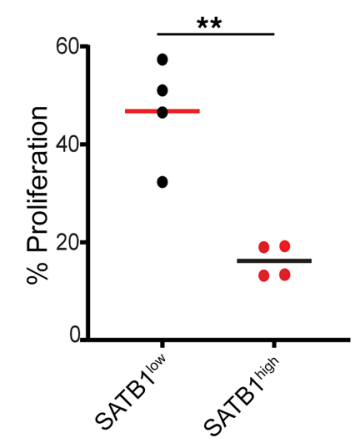


Figure 7. H3K9me $\mathrm{m}^{3}$ repression of SATB1 in malignant Sézary patient cells. (A) CD4+CD26-isolated T cells from peripheral blood apheresis of Sézary patients $(n=4)$ were cultured in R10 media with $100 \mathrm{U} / \mathrm{mL}$ human recombinant IL-2 and treated with increasing doses of SUV39H1/2 inhibitors chaetocin and F5446 (72 hours) as well as romidepsin (72 hours) and GSK126 (48 hours) versus the vehicle control (DMSO) prior to MTT analysis. $\mathrm{IC}_{50}$ values $(\mathrm{nM})$ were calculated for each patient sample for the respective treatments. (B) Summary of $\mathrm{IC}_{50}$ values for chaetocin, F5446, romidepsin, and GSK126 for each malignant sample $(n=4)$. One-way ANOVA with Tukey's multiple-comparison test: ${ }^{*} P<0.05$; ${ }^{* *} P \leq 0.01$. (C) RNA was extracted from primary $C D 4{ }^{+} C D 26$ - Sézary patient cells that were treated with chaetocin, F5446, romidepsin, or vehicle control (DMSO) for 24-36 hours, and SATB1 mRNA expression was quantified by q-PCR normalized to human TBP mRNA. Data pooled from 3 patient samples with 2 independent experiments shown $(n=6)$. Two-tailed Student's $t$ test: ${ }^{* * *} P \leq$ 0.0001 . (D) Chromatin immunoprecipitation quantified by real-time q-PCR with anti-H3K27 $\mathrm{me}^{3}$ (clone C36B11) or control IgC isotype immunoprecipitation from isolated $C D 4^{+} T$ cells from peripheral blood of Sézary patients $(n=2)$ calculated against $2.5 \%$ input values. Regions amplified at the predicted occupied region (approximately $4.8 \mathrm{~kb}$ )of the SATB1 promoter. Representative of 2 independent experiments. Two-tailed Student's $t$ test: ${ }^{*} P<0.05$; ${ }^{* *} P \leq 0.01$; ${ }^{* *} P \leq 0.001$. (E) Similar to $\mathbf{D}$ except with anti-H3K9me (Abcam, ab8898) at the approximately 5.6-kb region versus the control region $(n=2)$. Representative of 2 independent experiments. Two-tailed Student's $t$ test: ${ }^{*} P<0.05 ;{ }^{* *} P \leq 0.01$; ${ }^{* * *} P \leq 0.001$. (F) Primary Sézary CD4 ${ }^{+}$CD26 cells $(n=2)$ were transduced with retrovirus containing human SATB1 and sorted for GFP' and GFP- cells. Western blot was performed with antibodies against human p-STAT5 and STAT5 protein in cells endogenously expressing SATB1 versus cells with ectopic expression of SATB1 $(n=2)$. (G) Primary Sézary cells were labeled with CellTrace Violet and were serum starved for 24 hours prior to stimulation with anti-CD3/ anti-CD28 beads and cultured in complete medium with $100 \mathrm{U} / \mathrm{mL}$ rhIL-2 for 5 days. Proliferation was assessed by CellTrace Violet dilution using FACS for cells ectopically $(n=2)$ or endogenously $(n=2)$ expressing SATB1. Experiment was performed on 2 replicates per patient $(n=4)$. Two-tailed Student's $t$ test: ${ }^{* *} P \leq 0.01$.

AMPHO (ATCC, CRL-321) cells after transfection using Lipofectamine 3000 transfection reagent (Thermo Fisher Scientific, L3000001) following the manufacturer's recommendations with the pBMN-I-GFP vector containing human SATB1 ORF (GenScript Biotech) subcloned using the EcoRI and BamHI restriction sites. Viruses were collected in complete medium at 48 and 72 hours and filtered through a $40 \mu \mathrm{m}$ filter. Retronectin $(50 \mu \mathrm{g} / \mathrm{mL})$ in sterile PBS was used to coat non-tissue culture-treated 24 -well plates overnight at $4^{\circ} \mathrm{C}$. Wells were blocked with $2 \% \mathrm{BSA}$ in PBS for 15 minutes at room temperature and washed twice in sterile PBS. Plates were spun with $3 \mathrm{~mL}$ viral supernatant for 1.5 hours at $32^{\circ} \mathrm{C}$ and $2,000 \mathrm{~g}$. Supernatant was removed, leaving approximately $500 \mu \mathrm{L}$, and cells were added and spun for 10 minutes at $1500 \mathrm{rpm}, 32^{\circ} \mathrm{C}$, and cultured at $37^{\circ} \mathrm{C}$ in $5 \% \mathrm{CO}_{2}$ until GFP expression was detected at approximately 96 hours.

Cell lines and MTT assays. The HuT78 Sézary cell line (ATCC, TIB161) was cultured with Iscove's modified Dulbecco's medium (IMDM) with 20\% FBS and penicillin/streptomycin (Lonza, 17-602E). For viability analysis, HuT78 cells were seeded at 40,000 cells/well in triplicate in 96-well plates in the presence of vehicle control (ethanol or DMSO) or the EZH2 inhibitor GSK126 (Cayman Chemical, 15415), chaetocin (Abcam, ab144534), romidepsin (FK228, Depsipeptide; Selleck, S3020-5mg), F5446, and incubated at $37^{\circ} \mathrm{C}$ and $5 \% \mathrm{CO}_{2}$ for 48 or 72 hours. Ten microliters of $5 \mathrm{mg} / \mathrm{mL}$ MTT reagent (MilliporeSigma, M2128-1G) was added for 3 hours, followed by $100 \mu \mathrm{L}$ of lysis buffer
(44\% dimethylformamide, $2.2 \%$ acetic acid, $1.8 \% 1 \mathrm{~N}$ HCL, $17.7 \% \mathrm{w} / \mathrm{v}$ SDS, $\mathrm{NaOH}$ added to achieve $\mathrm{pH} 4.7$ ) overnight at $37^{\circ} \mathrm{C}$ and $5 \% \mathrm{CO}_{2}$. Results were measured at $560 \mathrm{~nm}$ on a microplate spectrophotometer (Bio-Rad Benchmark Plus). Jurkat (ATCC, TIB-152) cells were grown in RPMI plus 10\% FBS with penicillin/streptomycin, K562 (ATCC, CCL-243) cells were grown in IMDM plus 10\% FBS and penicillin/ streptomycin, and RAJI (ATCC, CCL-86) cells were grown in RPMI plus 10\% FBS and penicillin/streptomycin.

Antibodies and flow cytometry. We used the following anti-human antibodies and their clone numbers: $\mathrm{CD} 45$ (HI30), CD45RO (UCHL1), CD26 (BA5b), CD3 (OKT3), CD2 (RPA-2.10), CD5 (UCHT2), CD7 (CD7-6B7), TCR $\beta$ (IP26), CD52 (HI186), CD56 (5.1H11), CD57 (HCD57), and CCR4/CD194 (L291H4), all from Biolegend; and CD4 (SK3), CD8 (RPA-T8), SATB1 (14/satb1), and TCR $\gamma \delta$ (B1) from BD Biosciences. Human $\gamma$-globulin (MilliporeSigma, catalog G4386) was used for $\mathrm{Fc}$ receptor blockade.

Mouse antibodies and their clone numbers were as follows: CD45 (30-F11), CD5 (53-7.3), CD11b (M1/70), CD11c (N418), Ly6G (1A8), Ly6C (HK1.4), TCR $\beta$ (H57-597), TCR $\gamma \delta$ (GL3), NK1.1 (PK136), NKG2D (CX5), CD96 (6A6), CD25 (PC61), CD34 (MEC14.7), CCR4 (2G12), CCR7 (4B12), CCR6 (29-2L17), CD19 (6D5), CD62L (MEL-14) from Biolegend; MHCII (M5/114), CD2 (RM2-5), CD3e (145-2C11), CD4 (GK1.5), CD8 (53-6.7), and CD69 (H1.2F3) from BD Bioscience; CD44 (IM7) from Tonbo; and CCR10 (clone 248918) from R\&D Systems. Zombie yellow (BioLegend) was used as viability probe for all samples. Rat anti-CD16/anti-CD32 mAb (clone 2.4G2) was used for Fc receptor blockade.

For flow cytometry, all samples were run on a BD LSRII and sorted using a BD FACSAria SORP. Data were analyzed using FlowJo software v10.7. Intracellular staining was performed with a Transcription Factor Staining Buffer Set (Thermo Fisher Scientific).

The following antibodies were used for immunoblot analysis: anti-p-STAT5 (Tyr694; rabbit, Cell Signaling Technology, clone D47E7), STAT5 (rabbit, Cell Signaling Technology, clone D2O6Y), anti-mouse/human $\beta$-actin (Thermo Fisher Scientific, clone BA3R), and anti-Satb1 (BD Biosciences, clone 14).

When indicated, $\mathrm{CD}^{+} \mathrm{T}$ cells were isolated from mouse spleens using a Dynabeads Untouched Mouse T Cells Kit (Invitrogen, 11413D) from $5 \times 10^{6}$ total splenocytes following the manufacturer's recommendations.

CellTrace Violet assays and annexin $V$ staining. Cell tracing assays were performed using CellTrace Violet (Invitrogen, C34571) following the manufacturer's recommendations with $5 \mu \mathrm{M}$ stock concentration resuspended in DMSO, starting with $1 \times 10^{6}$ cells $/ \mathrm{mL}$ in sterile PBS. Cells were incubated at $37^{\circ} \mathrm{C}$ for 20 minutes protected from light. The reaction was quenched using complete cell culture medium at 5 times the reaction volume and incubated for 5 minutes, spun for 5 minutes at $4^{\circ} \mathrm{C}$ and $1500 \mathrm{rpm}$, and resuspended at $1 \times 10^{6}$ cells $/ \mathrm{mL}$ in fresh warm media and cultured for 5 days prior to FACS analysis. Cells were stained with DAPI (Invitrogen, D1306) to exclude dead cells from analysis.

Annexin V staining was performed with an APC Annexin V Apoptosis Detection Kit (BioLegend, 640932) and propidium iodide (PI) (BioLegend, 421301) for the identification of apoptotic and necrotic cells following the manufacturer's recommendations. Cells were washed twice with cold Cell Staining Buffer (BioLegend, 420201) and then resuspended in Annexin V Binding Buffer (BioLegend, 422201 ) at a concentration between $0.25 \times 10^{7}$ and $1.0 \times 10^{7}$ cells/ 
mL. Cells were then stained with APC-annexin V and PI. Cells were then incubated for 15 minutes at room temperature in the dark prior to resuspension in Annexin V Binding Buffer and FACS analysis. For flow cytometry, all samples were run on a BD LSRII and data were analyzed using FlowJo software.

Immunohistochemistry, Giemsa, and Western blot. Formalin-fixed, paraffin-embedded ventral and dorsal skin sections on charged slides (APEX-SAS, 3800080E) were stained using a Ready-to-use IHC/ ICC kit (BioVision, 501119739) following the manufacturer's instructions, following antigen retrieval (10 minutes boiling in citrate buffer) and deparaffination. Anti-CD3 was from Abcam (rabbit monoclonal, SP7). Slides were counterstained with hematoxylin (RICCA, 353032), dehydrated, and mounted with Permount (Thermo Fisher Scientific, SP15-100). For Giemsa staining, $5 \mu \mathrm{L}$ of noncoagulated blood smears were left to air dry and fixed in methanol 10 minutes at room temperature. Slides were then incubated in Giemsa (MilliporeSigma, 48900) 1:20 in PBS for 1 hour, washed in $\mathrm{ddH}_{2} \mathrm{O}$, and mounted with Permount. Histology slides were scanned using the Aperio ScanScope AT2 (Leica Biosystems) with a $200 \times / 0.8-N A$ objective lens with a doubler to image at $\times 400$ magnification. Image analysis for mouse slides was performed using an Aperio Positive Pixel Count v9.0 algorithm with the following thresholds to segment positive staining of various intensities: hue value $=0.1$, hue width $=0.5$, color saturation threshold $=0.04, \operatorname{IWP}($ high $)=220, \operatorname{IWP}($ low $)=\operatorname{Ip}($ High $)=175$; Ip $($ low $)=\operatorname{Isp}($ High $)=100, \operatorname{Isp}($ Low $)=0$.

For Western blot analysis, cells were lysed in RIPA buffer (Pierce) supplemented with protease inhibitors (Roche). Equal amounts of protein were quantified using the BCA Protein Assay kit (Pierce) and resolved by SDS-PAGE, blotted onto PVDF membranes (Millipore), and probed with the aforementioned antibodies.

$q$-PCR and ChIP. Real-time PCR amplification was carried out using SYBR Green on a 7500 Fast Real-Time PCR system (Applied Biosystems). Human SATB1 mRNA expression was quantified using the following primers, common to all human splicing variants: SATB1 forward, 5'-AGTGGGTACGCGATGAACTGAA-3' and SATB1 reverse, 5'-ATGCAGTCTTGGGGTCCTCTTC-3'. mRNA expression was normalized by GAPDH levels (primers GAPDH forward, 5 '-CCTGCACCACCAACTGCTTA-3' and GAPDH reverse, 5'-GTGATGGCATGGACTGTGGT-3') or TATA-binding protein (TBP) levels (primers TBP forward, 5'-CACGAACCACGGCACTGATT-3' and TBP reverse, 5'-TTTTCTTGCTGCCAGTCTGGAC-3'). The average of 3 independent analyses was calculated using the $\Delta \Delta$ threshold cycle (Ct) method and was normalized to the endogenous reference control gene GAPDH or TBP.

For ChIP experiments, the HuT78 cell line was seeded at 1.5 $\times 10^{6}$ cells/well in 6-well plates. ChIP assays were performed as we previously reported (7). Input and immunoprecipitated DNA were analyzed using SYBR Green in a real-time PCR machine (Applied Biosystems). Results shown for each ChIP condition were analyzed using the percentage input method; namely, the DNA recovered from the ChIP was divided by signals obtained from the input sample (signals calculated with $2.5 \%$ of the amount of chromatin used in the ChIP). Anti-H3K27me $\mathrm{e}^{3}$ (Cell Signaling Technology, rabbit, clone C36B11), H3K9 $\mathrm{me}^{3}$ (Abcam, rabbit, catalog ab8898), H3K27ac (Abcam, rabbit, catalog ab4729), and control IgG (Cell Signaling Technology, rabbit IgG) antibodies were used for ChIP. The following primers were used for promoter quantification: human SATB1 promoter $\mathrm{H} 3 \mathrm{~K} 27 \mathrm{me}^{3}$ forward, 5'-TTTCCCCTGTTGGTCTTCTGAGG-3' and reverse, 5'-TGGCCTTTTCCTATTGCTCCAGT-3'; human SATB1 promoter H3K9me ${ }^{3}$ forward, 5'-TCTATTGGGCAGGTGTGGTGG-3' and reverse, 5'-GCAGGGCTTCAACGGTCTTC-3'. Primers for control genomic regions were SATB1 control promoter forward, 5'-GAGGAGGAGGAAGATCAGAAGGC-3' and reverse, 5'-GTTTTGTCGGGGTTTCTGGGTTT-3'. Primers validating ChIP-seq experiment for $\mathrm{H} 3 \mathrm{~K} 9 \mathrm{me}^{3}$ region 1 forward, 5'-TTGCTTTTTCCTCCCATAG-3' and reverse, 5'-GAAATTTGTGGCCAACTACA-3'; H3K9me ${ }^{3}$ region 2 forward, 5'-AACCTGCCAGCTAAACAGTC-3' and reverse, 5'-GCCGACACTTAGGGAAAATA-3'; H3K9me ${ }^{3}$ region 2 primer 2 forward, 5'-AAGAAGGTCGGATAGTGCAG-3' and reverse, 5'-AGACTTGGGCCATAAAGCTA-3'; H3K9me ${ }^{3}$ region 2 primer 3 forward, 5'-AACAGGACCAGAGCAAAATC-3' and reverse, 5'-ACTAGGATTGGGAAACACCA-3'; H3K9 me ${ }^{3}$ region 3 forward, 5'-TTTCTACTGAAGGTCAAAGTGTTT-3' and reverse, 5'-CAGGTATGCATGTATAGGACAAA-3' (all sequences designed to give amplicons of $<200 \mathrm{bp}$ ). Amplification of target genes is shown as fold enrichment compared with that of irrelevant antibody controls.

Statistics. All statistical analyses were performed using GraphPad Prism 5.0 software. A 2-tailed Student's $t$ test was used for calculating differences between means of experimental groups. One-way ANOVA with Tukey's post hoc test was used for calculating differences between means using multiple comparisons between groups. Logrank (Mantel-Cox) test was used for survival curve analysis. $P$ values less than 0.05 were considered significant. Data are presented as mean \pm SEM for each group or measurement.

Study approval. This study conformed to approvals granted by the H. Lee Moffitt Cancer Center \& Research Institute research regulatory committees, including IRB approvals MCC 50175 (TCPL), MCC 50218 (T-LGL), and MCC 50229 (Sézary). Further, MCC 20032 for Sézary syndrome peripheral blood samples was acquired with informed written consent protocol MCC 14690. All experimental procedures involving vertebrate animals were conducted in accordance with the H. Lee Moffitt Cancer Center \& Research Institute IACUC approvals ISO0006598, ISO0006654, and IS00002583.

\section{Author contributions}

$\mathrm{CMH}$ and JPS designed, performed, and analyzed most of the experiments and cowrote the manuscript. The author order was chosen based on alphabetical order as the both authors contributed equally. TLC, KER, and KBS contributed to study design and processing of samples for transgenic mouse experiments, drug screening, and patient samples. LS contributed to the design of the study, patient samples, and provided intellectual support. JRCG oversaw and designed the study and experiments, analyzed data, and cowrote the manuscript. XY performed all biological analyses for experiments. KL provided F5446. KKP, CMA, RAC, SB, GM, JAM, LCS, $\mathrm{KL}, \mathrm{PCR}$, and JPI contributed to the overall data interpretation, provided intellectual input, and approved the final manuscript.

\section{Acknowledgments}

This study was supported by NIH grants R01CA240434, R01CA157664, and R01CA124515. KKP was supported by NIH T32CA009140 and an American Cancer Society Postdoctoral Fellowship (PF-18-041-1-LIB). LCS was supported by NIH U01 CA200495. This work has been supported in part by the 
Flow Cytometry Core, Molecular Genomics Core, and Analytic Microscopy Core facilities at the H. Lee Moffitt Cancer Center \& Research Institute, an NCI-designated Comprehensive Cancer Center (P30-CA076292).
Address correspondence to: Jose R. Conejo-Garcia, H. Lee Moffitt Cancer Center \& Research Institute, 12902 Magnolia Drive, Tampa, Florida 33612, USA. Phone: 813.745.8262; Email: Jose. Conejo-Garcia@Moffitt.org.
1. Beltran BE, et al. The neutrophil-lymphocyte ratio is prognostic in patients with early stage aggressive peripheral T cell lymphoma. $\mathrm{Br} \mathrm{J} \mathrm{Hae-}$ matol. 2019;184(4):650-653.

2. Rodd AL, et al. Current and emerging therapeutics for cutaneous T-cell lymphoma: histone deacetylase inhibitors. Lymphoma. 2012;2012:10

3. Kamstrup MR, et al. Notch1 as a potential therapeutic target in cutaneous T-cell lymphoma. Blood. 2010;116(14):2504-2512.

4. da Silva Almeida AC, et al. The mutational landscape of cutaneous T cell lymphoma and Sezary syndrome. Nat Genet. 2015;47(12):1465-70.

5 . Naik R, Galande S. SATB family chromatin organizers as master regulators of tumor progression. Oncogene. 2019;38(12):1989-2004.

6. Tesone AJ, et al. Satb1 overexpression drives tumor-promoting activities in cancer-associated dendritic cells. Cell Rep. 2016;14(7):1774-1786.

7. Ma Y, et al. CRISPR/Cas9 screens reveal EpsteinBarr virus-transformed B cell host dependency factors. Cell Host Microbe. 2017;21(5):580-591.e7.

8. Kitagawa Y, et al. Guidance of regulatory $T$ cell development by Satb1-dependent super-enhancer establishment. Nat Immunol. 2017;18(2):173-183.

9. Borghesi L. Hematopoiesis in steady-state versus stress: self-renewal, lineage fate choice, and the conversion of danger signals into cytokine signals in hematopoietic stem cells. J Immunol. 2014;193(5):2053-2058.

10. Fredholm $S$, et al. SATB1 in malignant $\mathrm{T}$ cells. J Invest Dermatol. 2018;138(8):1805-1815.

11. Murtaugh LC, et al. Notch signaling controls multiple steps of pancreatic differentiation. Proc Natl
Acad Sci U S A. 2003;100(25):14920-14925.

12. Vinay DS, Kwon BS. CD $11 \mathrm{c}^{+} \mathrm{CD}^{+} \mathrm{T}$ cells: twofaced adaptive immune regulators. Cell Immunol. 2010;264(1):18-22.

13. Seo SK, et al. 4-1BB-mediated immunotherapy of rheumatoid arthritis. Nat Med. 2004;10(10):1088-1094.

14. Payne KK, et al. BTN3A1 governs antitumor responses by coordinating $\alpha \beta$ and $\gamma \delta$ T cells. Science. 2020;369(6506):942-949.

15. Aghajani K, et al. Generation of CD4CreER(T) transgenic mice to study development of peripheral CD4-T-cells. Genesis. 2012;50(12):908-913.

16. Campbell JJ, et al. Sezary syndrome and mycosis fungoides arise from distinct $\mathrm{T}$-cell subsets: $\mathrm{a}$ biologic rationale for their distinct clinical behaviors. Blood. 2010;116(5):767-771.

17. Duvic M, et al. Phase $1 / 2$ study of mogamulizumab, a defucosylated anti-CCR4 antibody, in previously treated patients with cutaneous T-cell lymphoma. Blood. 2015;125(12):1883-1889.

18. Mindur JE, et al. Early treatment with antiVLA-4 mAb can prevent the infiltration and/or development of pathogenic CD $11 \mathrm{~b}^{+} \mathrm{CD} 4^{+} \mathrm{T}$ cells in the CNS during progressive EAE. PLoS One. 2014;9(6):e99068.

19. Wang Y, et al. Deficiency of SATB1 expression in Sezary cells causes apoptosis resistance by regulating FasL/CD95L transcription. Blood. 2011;117(14):3826-3835.

20. Jones PA, Baylin SB. The epigenomics of cancer. Cell. 2007;128(4):683-692.

21. Becker JS, et al. H3K9me3-dependent heterochromatin: barrier to cell fate changes. Trends Genet. 2016;32(1):29-41.
22. Bennett RL, Licht JD. Targeting epigenetics in cancer. Annu Rev Pharmacol Toxicol. 2018;58:187-207.

23. Rea S, et al. Regulation of chromatin structure by site-specific histone $\mathrm{H} 3$ methyltransferases. Nature. 2000;406(6796):593-599.

24. Collins PL, et al. The histone methyltransferase SETDB1 represses endogenous and exogenous retroviruses in B lymphocytes. Proc Natl Acad Sci US A. 2015;112(27):8367-8372.

25. Lu C, et al. SUV39H1 represses the expression of cytotoxic T-lymphocyte effector genes to promote colon tumor immune evasion. Cancer Immunol Res. 2019;7(3):414-427.

26. Kim YH, et al. Mogamulizumab versus vorinostat in previously treated cutaneous T-cell lymphoma (MAVORIC): an international, open-label, randomised, controlled phase 3 trial. Lancet Oncol. 2018;19(9):1192-1204.

27. Cai S, et al. SATB1 packages densely looped, transcriptionally active chromatin for coordinated expression of cytokine genes. Nat Genet. 2006;38(11):1278-1288.

28. Yasui D, et al. SATB1 targets chromatin remodelling to regulate genes over long distances. Nature. 2002;419(6907):641-645.

29. Zhang Q, et al. Activation of Jak/STAT proteins involved in signal transduction pathway mediated by receptor for interleukin 2 in malignant $\mathrm{T}$ lymphocytes derived from cutaneous anaplastic large T-cell lymphoma and Sezary syndrome. Proc Natl Acad Sci U S A . 1996;93(17):9148-9153.

30. Grzanka D, et al. Expression of special AT-rich sequence-binding protein 1 is an independent prognostic factor in cutaneous T-cell lymphoma. Oncol Rep. 2015;33(1):250-266. 